\title{
Teichmüller theory and collapse of flat manifolds
}

\author{
Renato G. Bettiol ${ }^{1}$ • Andrzej Derdzinski ${ }^{2}$ • \\ Paolo Piccione ${ }^{3}$
}

Received: 15 October 2017 / Accepted: 30 December 2017 / Published online: 18 January 2018

(C) Fondazione Annali di Matematica Pura ed Applicata and Springer-Verlag GmbH Germany, part of Springer Nature 2018

\begin{abstract}
We provide an algebraic description of the Teichmüller space and moduli space of flat metrics on a closed manifold or orbifold and study its boundary, which consists of (isometry classes of) flat orbifolds to which the original object may collapse. It is also shown that every closed flat orbifold can be obtained by collapsing closed flat manifolds, and the collapsed limits of closed flat 3-manifolds are classified.
\end{abstract}

Keywords Flat manifolds · Flat orbifolds · Teichmueller space · Moduli space · Collapse · Gromov-Hausdorff convergence

Mathematics Subject Classification 22E40 - 32G15 - 53C15 - 53C24 · 53C29 - 57M60 · $57 \mathrm{R} 18 \cdot 58 \mathrm{D} 17 \cdot 58 \mathrm{D} 27$

\section{Introduction}

A fundamental question in Riemannian geometry is whether there exist deformations of a given manifold that preserve certain curvature conditions and, if so, what is the nature of the

Renato G. Bettiol

rbettiol@math.upenn.edu

Andrzej Derdzinski

andrzej@math.ohio-state.edu

Paolo Piccione

piccione@ime.usp.br

1 Department of Mathematics, University of Pennsylvania, 209 South 33rd St, Philadelphia, PA 19104-6395, USA

2 Department of Mathematics, The Ohio State University, 231 W. 18th Avenue, Columbus, OH 43210, USA

3 Departamento de Matemática, Universidade de São Paulo, Rua do Matão 1010, São Paulo, SP 05508090, Brazil 
limiting spaces. In this paper, we study how flat manifolds and flat orbifolds can be deformed while keeping them flat and, in particular, how they collapse and what are the possible limits.

Lower curvature bounds (in triangle comparison sense) are preserved under GromovHausdorff limits, but the same does not hold for upper curvature bounds. Thus, the Gromov-Hausdorff limit of a sequence of closed flat manifolds is only, a priori, an Alexandrov space with nonnegative curvature. However, for instance, the Gromov-Hausdorff limit of a sequence of flat tori is a flat torus, by a classical compactness result of Mahler [27]. More generally, the only singularities that may arise collapsing general flat manifolds are the mildest possible, and any flat space with singularities of this type admits a smooth flat resolution:

Theorem A The Gromov-Hausdorff limit of a sequence of closed flat manifolds is a closed flat orbifold. Conversely, every closed flat orbifold is the Gromov-Hausdorff limit of a sequence of closed flat manifolds.

The formation of orbifold singularities in the collapse of smooth flat manifolds can be easily seen already in dimension 2. Consider flat Klein bottles as rectangles with the usual boundary identifications. The Gromov-Hausdorff limit obtained by shrinking the lengths of a pair of opposite sides is either $S^{1}$ or a closed interval (a flat 1-orbifold), depending on whether the identification of these sides preserves or reverses orientation.

A simple diagonal argument, combined with Theorem A, implies that the collection of closed flat orbifolds is closed in the Gromov-Hausdorff topology. In light of the fact that every object in this collection is the limit of smooth flat manifolds, it would be interesting to determine whether every orbifold with $\mathrm{sec} \geq 0$ is the limit of manifolds with sec $\geq 0$; see Remark 2.1. An important and currently open question is whether every finite-dimensional Alexandrov space with curv $\geq K$ is the limit of smooth manifolds with sec $\geq K$. In this context, recall that Alexandrov spaces of dimensions 3 and 4 are homeomorphic to orbifolds [17,21].

Analyzing deformations and limits of flat orbifolds leads to investigating the moduli space $\mathcal{M}_{\text {flat }}(\mathcal{O})$ of flat metrics on a fixed flat orbifold $\mathcal{O}$ and its ideal boundary. The nature of this moduli space is very reminiscent of the classical Teichmüller theory for hyperbolic surfaces, in that $\mathcal{M}_{\text {flat }}(\mathcal{O})$ is the quotient of a Teichmüller space $\mathcal{T}_{\text {flat }}(\mathcal{O})$, diffeomorphic to an open ball, under the action of a (discrete) mapping class group. This fits the picture of a deformation theory for geometric structures of much larger scope pioneered by Thurston [33] and further developed in [3,18]; see Sect. 4.3. Around 45 years ago, Wolf [34] identified the moduli space $\mathcal{M}_{\text {flat }}(M)$ of flat metrics on a flat manifold $M$. However, even in this special case, a systematic and unified treatment of the Teichmüller theory of flat manifolds does not seem to be available in the literature, despite some scattered results, e.g., [24,25]. Toward this goal, we establish the following algebraic description of the Teichmüller space of flat metrics on a flat orbifold, which provides a straightforward method to compute it:

Theorem B Let $\mathcal{O}$ be a closed flat orbifold, and denote by $W_{i}, 1 \leq i \leq l$, the isotypic components of the orthogonal representation of its holonomy group. Each $W_{i}$ consists of $m_{i}$ copies of the same irreducible representation, and we write $\mathbb{K}_{i}$ for $\mathbb{R}, \mathbb{C}$, or $\mathbb{H}$, according to this irreducible being of real, complex, or quaternionic type. The Teichmüller space $\mathcal{T}_{\text {flat }}(\mathcal{O})$ is diffeomorphic to:

$$
\mathcal{T}_{\text {flat }}(\mathcal{O}) \cong \prod_{i=1}^{l} \frac{\mathrm{GL}\left(m_{i}, \mathbb{K}_{i}\right)}{\mathrm{O}\left(m_{i}, \mathbb{K}_{i}\right)},
$$


where $\mathrm{GL}(m, \mathbb{K})$ is the group of $\mathbb{K}$-linear automorphisms of $\mathbb{K}^{m}$ and $\mathrm{O}(m, \mathbb{K})$ stands for $\mathrm{O}(m)$, $\bigcup(m)$, or $\mathrm{Sp}(m)$, when $\mathbb{K}$ is, respectively, $\mathbb{R}, \mathbb{C}$, or $\mathbb{H}$. In particular, $\mathcal{T}_{\text {flat }}(\mathcal{O})$ is real analytic and diffeomorphic to $\mathbb{R}^{d}$.

The dimension $d=\operatorname{dim} \mathcal{T}_{\text {flat }}(\mathcal{O})$ is easily computed as the sum of the dimensions $d_{i} \geq 1$ of the factors $\mathrm{O}\left(m_{i}, \mathbb{K}_{i}\right) \backslash \mathrm{GL}\left(m_{i}, \mathbb{K}_{i}\right) \cong \mathbb{R}^{d_{i}}, 1 \leq i \leq l$, which are given by:

$$
d_{i}= \begin{cases}\frac{1}{2} m_{i}\left(m_{i}+1\right), & \text { if } \quad \mathbb{K}_{i}=\mathbb{R}, \\ m_{i}^{2}, & \text { if } \quad \mathbb{K}_{i}=\mathbb{C}, \\ m_{i}\left(2 m_{i}-1\right), & \text { if } \quad \mathbb{K}_{i}=\mathbb{H}\end{cases}
$$

In particular, since the holonomy representation of a flat manifold is reducible [22], see Theorem 2.4, it follows that $l \geq 2$, and hence, $d \geq 2$. This implies the following:

\section{Corollary C Every flat manifold admits nonhomothetic flat deformations.}

The situation is different for flat orbifolds, which can be rigid. Examples of orbifolds with irreducible holonomy representation, i.e., $l=1$, which consequently admit no nonhomothetic flat deformations, already appear in dimension 2, for instance, flat equilateral triangles; see Sect. 5.3 for more examples.

Since flat orbifolds are locally isometric to Euclidean spaces, the most interesting aspects of their geometry are clearly global. Thus, it is not surprising that issues related to holonomy play a central role in developing this Teichmüller theory. As an elementary case illustrating Theorem B, consider the complete absence of holonomy: flat $n$-dimensional tori $T^{n}$ can be realized as parallelepipeds spanned by linearly independent vectors $v_{1}, \ldots, v_{n} \in \mathbb{R}^{n}$, with opposite faces identified. Flat metrics on $T^{n}$ correspond to different choices of $v_{1}, \ldots, v_{n}$, up to ambiguities arising from rigid motions in $\mathbb{R}^{n}$, or relabelings and subdivisions of the parallelepiped into smaller pieces with boundary identifications. More precisely, it is not difficult to see that $\mathcal{M}_{\text {flat }}\left(T^{n}\right)=\mathrm{O}(n) \backslash \mathrm{GL}(n, \mathbb{R}) / \mathrm{GL}(n, \mathbb{Z})$. In this case, $\mathcal{T}_{\text {flat }}\left(T^{n}\right)=\mathrm{O}(n) \backslash \mathrm{GL}(n, \mathbb{R}) \cong \mathbb{R}^{n(n+1) / 2}$ is the space of inner products on $\mathbb{R}^{n}$, and $\mathcal{M}_{\text {flat }}\left(T^{n}\right)=\mathcal{T}_{\text {flat }}\left(T^{n}\right) / \mathrm{GL}(n, \mathbb{Z})$; see Sect. 5.1 for details.

Isometry classes of collapsed limits of $T^{n}$ correspond to points in the ideal boundary of $\mathcal{M}_{\text {flat }}\left(T^{n}\right)$. A more tangible object is the ideal boundary of $\mathcal{T}_{\text {flat }}\left(T^{n}\right)$, formed by positive semidefinite $n \times n$ matrices and stratified by their rank $k$, with $0 \leq k<n$, which in a sense correspond to the $k$-dimensional flat tori $T^{k}$ to which $T^{n}$ can collapse. Nevertheless, we warn the reader that the Gromov-Hausdorff distance does not extend continuously to this boundary. For instance, collapsing the 2-dimensional square torus along a line of slope $p / q$, with $p, q \in \mathbb{Z}, \operatorname{gcd}(p, q)=1$, produces as Gromov-Hausdorff limit the circle $S^{1}$ of length $\left(p^{2}+q^{2}\right)^{-1 / 2}$, while collapsing it along any nearby line with irrational slope produces as limit a single point.

Recall that there are precisely 17 affine classes of flat 2-orbifolds, corresponding to the 17 wallpaper groups, see, for instance, [13]. The underlying topological space of $\mathcal{O}$ is a 2manifold $|\mathcal{O}|$, possibly with boundary, namely the disk $D^{2}$, sphere $S^{2}$, real projective plane $\mathbb{R} P^{2}$, torus $T^{2}$, Klein bottle $K^{2}$, cylinder $S^{1} \times I$, or Möbius band $M^{2}$. The singularities that occur in the interior are cone points, labeled with a positive integer $n \in \mathbb{N}$, specifying that the local group is the cyclic group $\mathbb{Z}_{n} \subset \mathrm{SO}(2)$. Singularities that occur on the boundary are corner reflectors, labeled by a positive integer $m \in \mathbb{N}$, specifying that the local group is the dihedral group $D_{m} \subset \mathrm{O}(2)$ of $2 m$ elements. Following Davis [13], if a 2-orbifold $\mathcal{O}$ has $\ell$ cone points labeled $n_{1}, \ldots, n_{\ell}$, and $k$ corner reflectors labeled $m_{1}, \ldots, m_{k}$, then it is denoted $|\mathcal{O}|\left(n_{1}, \ldots, n_{\ell} ; m_{1}, \ldots, m_{k}\right)$. 
By direct inspection, we verify that only 10 out of the 17 flat orbifolds of dimension 2 (see Table 1) arise as Gromov-Hausdorff limits of closed flat 3-manifolds:

Theorem D The Gromov-Hausdorfflimit of a sequence of closed flat 3-manifolds is either a closed flat 3-manifold, or one of the following collapsed cases: point, closed interval, circle, 2-torus, Klein bottle, Möbius band, cylinder, disk with singularities $D^{2}(4 ; 2), D^{2}(3 ; 3)$, or $D^{2}(2,2 ;)$, sphere with singularities $S^{2}(3,3,3 ;)$ or $S^{2}(2,2,2,2$;), or the real projective plane with singularities $\mathbb{R} P^{2}(2,2 ;)$.

The remaining 7 flat 2-orbifolds must arise as Gromov-Hausdorff limits of flat manifolds of dimensions $\geq 4$. For example, it is easy to see that a flat rectangle $D^{2}(; 2,2,2,2)$ can be realized as the collapsed limit of a product $K^{2} \times K^{2}$ of Klein bottles. However, there does not seem to be a readily available method to decide what is the lowest dimension $N(\mathcal{O})$ of a flat manifold that collapses to a given flat orbifold $\mathcal{O}$. Heuristically, we expect that an upper bound for $N(\mathcal{O})$ could be derived from the structure of the singular set of $\mathcal{O}$. For instance, adding one dimension for each cone point, one could expect to replace the rotation element that fixes the cone point with a screw motion having a translation component in this new direction, which hence acts freely and resolves that conical singularity.

Another method to estimate $N(\mathcal{O})$, following the proof of Theorem A, is to estimate the lowest dimension of a flat manifold with prescribed holonomy group $H$ isomorphic to the holonomy group of $\mathcal{O}$. Such a flat manifold always exists by a result of Auslander and Kuranishi [2], see Theorem 2.3, and determining its lowest dimension is a well-known open question, see Szczepański [31, Problem 1]. An answer to this problem, and consequently an upper estimate for $N(\mathcal{O})$, is available when the group $H$ is cyclic, an elementary abelian $p$ group, dihedral, semidihedral, a generalized quaternion group, or a simple group $\operatorname{PSL}(2, p)$ with $p$ prime. According to Szczepański [31], the difficulty in establishing more comprehensive results in this direction is related to the difficulty in computing the second group cohomology of $H$ with special coefficients.

There are several other questions related to the results in this paper, two of which we would like to emphasize. The first is to characterize algebraically which isotypic components of the holonomy group of a flat manifold produce as Gromov-Hausdorff limit another flat manifold (instead of a nonsmooth flat orbifold) when collapsed. The second is to what extent the above results generalize to the class of almost flat manifolds.

This paper is organized as follows. Preliminary facts about closed flat manifolds and flat orbifolds are recalled in Sect. 2, including the Bieberbach Theorems and the classification of flat 2-orbifolds. Limits of closed flat manifolds are studied in Sect. 3, which contains the proof of Theorem A. Section 4 deals with Teichmüller spaces and moduli spaces of flat metrics, establishing the algebraic characterization given by Theorem B. Examples of Teichmüller spaces that can be computed by applying Theorem B are discussed in Sect. 5. Section 6 contains the classification of limits of flat 3-manifolds and the proof of Theorem D.

\section{Flat manifolds and flat orbifolds}

In this section, we recall basic facts about closed flat manifolds and flat orbifolds.

\subsection{Orbifolds}

A Riemannian orbifold $\mathcal{O}$ is a metric space which is locally isometric to orbit spaces of isometric actions of finite groups on Riemannian manifolds. Geometric properties, such as 
curvature, may be defined via these local isometries. Following Thurston [33], $\mathcal{O}$ is called good if it is globally isometric to such an orbit space. Any orbifold $\mathcal{O}$ has a universal orbifold covering $\widetilde{\mathcal{O}}$, with a discrete isometric action by deck transformations of its orbifold fundamental group $\pi_{1}^{\text {orb }}(\mathcal{O})$. The orbifold $\mathcal{O}$ has nonempty boundary (as an Alexandrov space) if and only if $\pi_{1}^{\mathrm{orb}}(\mathcal{O})$ contains a reflection, that is, an involution with fixed point set of codimension 1.

Every Riemannian orbifold $\mathcal{O}$ of dimension $n$ has a frame bundle $\operatorname{Fr}(\mathcal{O})$, which is a (smooth) Riemannian manifold with an almost free isometric $\mathrm{O}(n)$-action whose orbit space is $\operatorname{Fr}(\mathcal{O}) / \mathrm{O}(n) \cong \mathcal{O}$. In particular, it follows that every Riemannian orbifold is the (continuous) Gromov-Hausdorff limit of Riemannian manifolds $\left\{\left(\operatorname{Fr}(\mathcal{O}), g_{t}\right)\right\}_{t \geq 0}$, where $g_{t}$ is the Cheeger deformation of some invariant metric with respect to the $\mathrm{O}(n)$-action, see $[1, \S 6.1]$. For details on the basic geometry and topology of orbifolds, see [7,13,26,33].

Remark 2.1 At first sight, since Cheeger deformations preserve sec $\geq 0$, the above facts may seem to provide an approach to solving the question in the Introduction about realizing an orbifold $\mathcal{O}$ with sec $\geq 0$ as a limit of manifolds with sec $\geq 0$. However, it is in general difficult to endow $\operatorname{Fr}(\mathcal{O})$ with $\mathrm{sec} \geq 0$. This problem is related to the well-known converse question to the Soul Theorem of Gromoll and Meyer, of which vector bundles over closed manifolds with $\sec \geq 0$ admit metrics with $\sec \geq 0$.

In the very special case of a spherical orbifold $\mathcal{O}=S^{n} / \Gamma$, such as the spherical suspension of $\mathbb{R} P^{2}$, the frame bundle $\operatorname{Fr}(\mathcal{O})=\mathrm{O}(n+1) / \Gamma$ clearly admits sec $\geq 0$. Thus, Cheeger deformations allow to approximate $\mathcal{O}$ by manifolds with sec $\geq 0$. However, it is unclear whether that can be done keeping the same optimal lower curvature bound $\mathrm{sec} \geq 1$ of the limit $\mathcal{O}$.

\subsection{Bieberbach theorems}

A discrete group $\pi$ of isometries of $\mathbb{R}^{n}$ is called crystallographic if it has compact fundamental domain in $\mathbb{R}^{n}$, so that $\mathcal{O}=\mathbb{R}^{n} / \pi$ is a closed flat orbifold. A torsion-free crystallographic group $\pi$ is called a Bieberbach group, and in this case the action of $\pi$ on $\mathbb{R}^{n}$ is free, so $M=\mathbb{R}^{n} / \pi$ is a closed flat manifold. Conversely, it is well known that, by the Killing-Hopf Theorem, if a closed manifold $M$ of dimension $n \geq 2$ carries a flat Riemannian metric, then its universal covering is $\mathbb{R}^{n}$ and its fundamental group is isomorphic to a Bieberbach group. Similarly, by a result of Thurston [33] (see [28]), if a closed Riemannian orbifold $\mathcal{O}$ of dimension $n \geq 2$ is flat, then it is good, its universal orbifold covering is $\mathbb{R}^{n}$, and its orbifold fundamental group is isomorphic to a crystallographic group. In all of these cases, we denote by $g_{\pi}$ the flat metric for which the quotient map $\left(\mathbb{R}^{n}, g_{\text {flat }}\right) \rightarrow\left(\mathbb{R}^{n} / \pi, g_{\pi}\right)$ is a Riemannian covering.

Denote by $\operatorname{Aff}\left(\mathbb{R}^{n}\right):=\mathrm{GL}(n) \ltimes \mathbb{R}^{n}$ the group of affine transformations of $\mathbb{R}^{n}$, and by Iso $\left(\mathbb{R}^{n}\right):=\mathrm{O}(n) \ltimes \mathbb{R}^{n}$ be the subgroup of rigid motions, that is, isometries of the Euclidean space $\left(\mathbb{R}^{n}, g_{\text {flat }}\right)$. We write elements of $\operatorname{Aff}\left(\mathbb{R}^{n}\right)$ and $\operatorname{Iso}\left(\mathbb{R}^{n}\right)$ as pairs $(A, v)$, with $A \in \mathrm{GL}(n)$ or $\mathrm{O}(n)$, and $v \in \mathbb{R}^{n}$. The group operation is given by

$$
(A, v) \cdot(B, w)=(A B, A w+v),
$$

and clearly $(A, v)^{-1}=\left(A^{-1},-A^{-1} v\right)$. The natural action of these groups on $\mathbb{R}^{n}$ is given by $(A, v) \cdot w=A w+v$.

Consider the projection homomorphism:

$$
\mathfrak{r}: \operatorname{Aff}\left(\mathbb{R}^{n}\right) \longrightarrow \operatorname{GL}(n), \quad \mathfrak{r}(A, v)=A .
$$


Given a crystallographic group $\pi \subset \operatorname{Iso}\left(\mathbb{R}^{n}\right)$, its image $H_{\pi}:=\mathfrak{r}(\pi)$ is called the holonomy of $\pi$. Let $L_{\pi}$ denote the kernel of the restriction of $\mathfrak{r}$ to $\pi$, so that we have a short exact sequence

$$
1 \longrightarrow L_{\pi} \longrightarrow \pi \longrightarrow H_{\pi} \longrightarrow 1 \text {. }
$$

The group $L_{\pi}$, which consists of elements of the form (Id, $v$ ), with $v \in \mathbb{R}^{n}$, is the maximal normal abelian subgroup of $\pi$ and is naturally identified with a subgroup of $\mathbb{R}^{n}$.

The Bieberbach Theorems [5,6], see also [9,10,32], provide the essential facts about the groups in (2) and have equivalent algebraic and geometric formulations:

Bieberbach Theorems (Algebraic version) The following hold:

I. If $\pi \subset \operatorname{Iso}\left(\mathbb{R}^{n}\right)$ is a crystallographic group, then $H_{\pi}$ is finite and $L_{\pi}$ is a lattice that spans $\mathbb{R}^{n}$.

II. Let $\pi, \pi^{\prime} \subset \operatorname{Iso}\left(\mathbb{R}^{n}\right)$ be crystallographic subgroups. If there exists an isomorphism $\phi: \pi \rightarrow \pi^{\prime}$, then $\phi$ is a conjugation in $\operatorname{Aff}\left(\mathbb{R}^{n}\right)$, i.e., there exists $(A, v) \in \operatorname{Aff}\left(\mathbb{R}^{n}\right)$ such that $\phi(B, w)=(A, v) \cdot(B, w) \cdot(A, v)^{-1}$ for all $(B, w) \in \pi$.

III. For all $n$, there are only finitely many isomorphism classes of crystallographic subgroups of $\operatorname{Iso}\left(\mathbb{R}^{n}\right)$.

Bieberbach Theorems (Geometric version) The following hold:

I. If $(\mathcal{O}, g)$ is a closed flat orbifold with $\operatorname{dim} \mathcal{O}=n$, then $(\mathcal{O}, g)$ is covered by a flat torus of dimension $n$, and the covering map is a local isometry.

II. If $\mathcal{O}$ and $\mathcal{O}^{\prime}$ are closed flat orbifolds of the same dimension with isomorphic fundamental groups, then $\mathcal{O}$ and $\mathcal{O}^{\prime}$ are affinely equivalent.

III. For all n, there are only finitely many affine equivalence classes of closed flat orbifolds of dimension $n$.

The list of (affine equivalence classes of) closed flat orbifolds of dimension $n$, which, by the above, is in bijective correspondence with the list of (affine conjugate classes of) crystallographic groups in Iso $\left(\mathbb{R}^{n}\right)$, is known for some small values of $n$ :

- If $n=2$, there are 17 examples, corresponding to the 17 wallpaper groups. They are listed in Table 1, using the notation for cone points and corner reflectors as in the Introduction (following Davis [13]). The corresponding wallpaper groups $\pi$ are identified by their crystallographic notation, followed by Conway's notation [12] in parenthesis, and their holonomy group $H_{\pi}$ is also indicated;

- If $n=3$, there are 219 examples, corresponding to the 219 space groups classified (independently) by Barlow, Fedorov, and Schönflies, in the 1890s;

- If $n=4$, there are 4,783 examples classified by Brown et al. [8];

- If $n=5$ and $n=6$, there are, respectively, 222,018 and 28,927,922 examples, obtained with the computer program CARAT, see Plesken and Schulz [30].

The sublist of (affine equivalence classes of) closed flat manifolds of dimension $n$ is also known for the above values of $n$ and is considerably shorter:

- If $n=2$, there are 2 examples: the torus $T^{2}$ and the Klein bottle $K^{2}$;

- If $n=3$, there are 10 examples, obtained by Hantzsche and Wendt [20], see Wolf [35, Thm. 3.5.5, 3.5.9];

- If $n=4$, there are 74 examples, obtained by Calabi, see Wolf [35, Sec. 3.6];

- If $n=5$ and $n=6$, there are, respectively, 1060 and 38,746 examples, obtained with the computer program CARAT, see Cid and Schulz [11]. 
Table 1 Flat 2-dimensional orbifolds

\begin{tabular}{|c|c|c|c|c|c|}
\hline \# & Orbifold $\mathbb{R}^{2} / \pi$ & Topology & Geometry & $\pi$ & $H_{\pi}$ \\
\hline (1) & $D^{2}(; 3,3,3)$ & $D^{2}$ & Equilateral triangle & p3m1 (*333) & $D_{3}$ \\
\hline (2) & $D^{2}(; 2,3,6)$ & $D^{2}$ & Triangle with angles $\frac{\pi}{2}, \frac{\pi}{3}, \frac{\pi}{6}$ & p6m $(* 632)$ & $D_{6}$ \\
\hline (3) & $D^{2}(; 2,4,4)$ & $D^{2}$ & Triangle with angles $\frac{\pi}{2}, \frac{\pi}{4}, \frac{\pi}{4}$ & $\mathrm{p} 4 \mathrm{~m}(* 442)$ & $D_{4}$ \\
\hline (4) & $D^{2}(; 2,2,2,2)$ & $D^{2}$ & Rectangle & $\operatorname{pmm}(* 2222)$ & $D_{2}$ \\
\hline (5) & $D^{2}(2 ; 2,2)$ & $D^{2}$ & $\begin{array}{l}\text { Quotient of a square by group } \mathbb{Z}_{2} \\
\text { generated by the rotation of } \pi \\
\text { around its center }\end{array}$ & $\mathrm{cmm}\left(2^{*} 22\right)$ & $D_{2}$ \\
\hline (6) & $D^{2}(4 ; 2)$ & $D^{2}$ & $\begin{array}{l}\text { Quotient of a square by group } \mathbb{Z}_{4} \\
\text { generated by the rotation of } \frac{\pi}{2} \\
\text { around its center }\end{array}$ & $\mathrm{p} 4 \mathrm{~g}\left(4^{*} 2\right)$ & $D_{4}$ \\
\hline (7) & $D^{2}(3 ; 3)$ & $D^{2}$ & $\begin{array}{l}\text { Quotient of an equilateral triangle by } \\
\text { group } \mathbb{Z}_{3} \text { generated by the rotation } \\
\text { of } \frac{\pi}{3} \text { around its center }\end{array}$ & p31m $\left(3^{*} 3\right)$ & $D_{3}$ \\
\hline (8) & $D^{2}(2,2 ;)$ & $D^{2}$ & $\begin{array}{l}\text { Half pillowcase: quotient of } \\
S^{2}(2,2,2,2 ;) \text { by reflection about } \\
\text { the equator }\end{array}$ & $\operatorname{pmg}\left(22^{*}\right)$ & $D_{2}$ \\
\hline (9) & $S^{2}(2,2,2,2 ;)$ & $S^{2}$ & $\begin{array}{l}\text { Pillowcase: Alexandrov double of } \\
\text { rectangle }\end{array}$ & p2 (2222) & $D_{2}$ \\
\hline (10) & $S^{2}(3,3,3 ;)$ & $S^{2}$ & $\begin{array}{l}\text { 333-turnover: Alexandrov double of } \\
\qquad D^{2}(; 3,3,3)\end{array}$ & p3 (333) & $\mathbb{Z}_{3}$ \\
\hline (11) & $S^{2}(2,3,6 ;)$ & $S^{2}$ & $\begin{array}{l}\text { 236-turnover: Alexandrov double of } \\
\qquad D^{2}(; 2,3,6)\end{array}$ & p6 (632) & $\mathbb{Z}_{6}$ \\
\hline (12) & $S^{2}(2,4,4 ;)$ & $S^{2}$ & $\begin{array}{l}\text { 244-turnover: Alexandrov double of } \\
\qquad D^{2}(; 2,4,4)\end{array}$ & p4 (442) & $D_{4}$ \\
\hline (13) & $\mathbb{R} P^{2}(2,2 ;)$ & $\mathbb{R} P^{2}$ & $\begin{array}{l}\text { Quotient of } S^{2}(2,2,2,2 ;) \text { by } \\
\text { antipodal map }\end{array}$ & $\operatorname{pgg}(22 \times)$ & $D_{2}$ \\
\hline (14) & $T^{2}$ & $T^{2}$ & 2-torus & p1 (o) & $\{1\}$ \\
\hline (15) & $K^{2}$ & $K^{2}$ & Klein bottle & $\operatorname{pg}(\times \times)$ & $\mathbb{Z}_{2}$ \\
\hline (16) & $S^{1} \times I$ & $S^{1} \times I$ & Cylinder & $\operatorname{pm}(* *)$ & $\mathbb{Z}_{2}$ \\
\hline (17) & $M^{2}$ & $M^{2}$ & Möbius band & $\mathrm{cm}\left({ }^{*} \times\right)$ & $\mathbb{Z}_{2}$ \\
\hline
\end{tabular}

\subsection{Holonomy group}

Let $\pi \subset \operatorname{Iso}\left(\mathbb{R}^{n}\right)$ be a crystallographic group and consider its holonomy group $H_{\pi}$.

Lemma 2.2 The lattice $L_{\pi} \subset \mathbb{R}^{n}$ is invariant under the orthogonal action of $H_{\pi}$.

Proof This is an easy consequence of normality of $L_{\pi}$ in $\pi$. Namely, given $w \in L_{\pi}$ and $A \in H_{\pi}$, let $v \in \mathbb{R}^{n}$ be such that $(A, v) \in \pi$. Then, by normality, there exists $w^{\prime} \in L_{p}$ such that $(A, A w+v)=(A, v) \cdot(\mathrm{Id}, w)=\left(\operatorname{Id}, w^{\prime}\right) \cdot(A, v)=\left(A, w^{\prime}+v\right)$, i.e., $A w=w^{\prime}$. Thus, $H_{\pi}\left(L_{\pi}\right) \subset L_{\pi}$.

If $\pi \subset \operatorname{Iso}\left(\mathbb{R}^{n}\right)$ is a Bieberbach group, then the orthogonal representation of its holonomy $H_{\pi}$ on $\mathbb{R}^{n}$ is identified with the holonomy representation of the flat manifold $M=\mathbb{R}^{n} / \pi$. In particular, notice that any two flat metrics on $M$ have isomorphic holonomy groups. 
Furthermore, the Betti numbers of $M$ are given by $b_{k}(M)=\operatorname{dim}\left(\wedge^{k} \mathbb{R}^{n}\right)^{H_{\pi}}$, that is, the dimension of the subspace of $\wedge^{k} \mathbb{R}^{n}$ fixed by the induced orthogonal representation of $H_{\pi}$.

The closed flat manifold $M=\mathbb{R}^{n} / \pi$ can also be seen as the orbit space of a free isometric action of $H_{\pi}$ on the flat torus $\mathbb{R}^{n} / L_{\pi}$. Namely, since $L_{\pi}$ is normal in $\pi$, the projection map $\mathbb{R}^{n} / L_{\pi} \rightarrow M$ is a regular (Riemannian) covering whose group of deck transformations is identified with $\pi / L_{\pi} \cong H_{\pi}$. In particular, it follows that $\operatorname{Vol}\left(M, g_{\pi}\right)=$ $\left|H_{\pi}\right|^{-1} \operatorname{Vol}\left(\mathbb{R}^{n} / L_{\pi}\right)$.

The free action of $H_{\pi}$ on $\mathbb{R}^{n} / L_{\pi}$ with orbit space $M$ can be described explicitly. First, note that since $L_{\pi}$ is $H_{\pi}$-invariant (Lemma 2.2), the natural action of $H_{\pi}$ on $\mathbb{R}^{n}$ descends to an action of $H_{\pi}$ on the torus $\mathbb{R}^{n} / L_{\pi}$. Given $A \in H_{\pi}$, denote by $\bar{A}: \mathbb{R}^{n} / L_{\pi} \rightarrow \mathbb{R}^{n} / L_{\pi}$ the induced map, and let $v \in \mathbb{R}^{n}$ be such that $(A, v) \in \pi$. Such $v$ is unique, up to translations in $L_{\pi}$, for if $v, v^{\prime} \in \mathbb{R}^{n}$ are such that $(A, v),\left(A, v^{\prime}\right) \in \pi$, then $(A, v) \cdot\left(A, v^{\prime}\right)^{-1}=\left(\operatorname{Id}, v-v^{\prime}\right) \in$ $L_{\pi}$, i.e., $v-v^{\prime} \in L_{\pi}$. We thus have a map $H_{\pi} \ni A \mapsto v_{A} \in \mathbb{R}^{n} / L_{\pi}$, defined by $v_{A}:=v+L_{\pi}$, where $(A, v) \in \pi$. Clearly, $v_{A \cdot A^{\prime}}=\bar{A}\left(v_{A^{\prime}}\right)+v_{A}$ for all $A, A^{\prime} \in H_{\pi}$, where + is the group operation on $\mathbb{R}^{n} / L_{\pi}$. With this notation, the free action of $H_{\pi}$ on $\mathbb{R}^{n} / L_{\pi}$ is:

$$
A \cdot \bar{x}:=\bar{A}(\bar{x})+v_{A}, \quad A \in H_{\pi}, \bar{x} \in \mathbb{R}^{n} / L_{\pi} .
$$

We conclude this with two very useful results about holonomy groups $H_{\pi}$. First, by a celebrated theorem of Auslander and Kuranishi [2], see also Wolf [35], there are no obstructions on $H_{\pi}$; more precisely:

Theorem 2.3 Any finite group is the holonomy group $H_{\pi}$ of a closed flat manifold.

Second, Hiss and Szczepański [22] established the following remarkable result:

Theorem 2.4 For any Bieberbach group $\pi \subset \operatorname{Iso}\left(\mathbb{R}^{n}\right)$, the orthogonal action of $H_{\pi}$ on $\mathbb{R}^{n}$ is reducible.

Remark 2.5 The proof of Theorem 2.4 is rather involved; however, it is worth observing that it becomes elementary if $H_{\pi}$ has nontrivial center. Namely, since the action of $\pi$ on $\mathbb{R}^{n}$ is free, given $(A, v) \in \pi,(A, v) \neq(\mathrm{Id}, 0)$, there does not exist $x \in \mathbb{R}^{n}$ such that $A x+v=x$, i.e., $(A-\mathrm{Id}) x=-v$. Thus, $(A-\mathrm{Id})$ is not invertible; hence, 1 is an eigenvalue of $A$. This gives a nontrivial orthogonal decomposition $\mathbb{R}^{n}=\operatorname{ker}(A-\mathrm{Id}) \oplus \operatorname{im}(A-\mathrm{Id})$, and $\operatorname{ker}(A-\mathrm{Id})$ is clearly $H_{\pi}$-invariant if $A \in Z\left(H_{\pi}\right)$.

The hypothesis that $\pi$ is torsion-free is essential in Theorem 2.4. In fact, it is easy to find crystallographic groups $\pi \subset \operatorname{Iso}\left(\mathbb{R}^{n}\right)$ whose holonomy $H_{\pi}$ acts irreducibly on $\mathbb{R}^{n}$, see Sect. 5.3 for examples with $n=2$.

\section{Sequences of flat manifolds}

In this section, we analyze sequences of closed flat manifolds, proving Theorem A.

\subsection{Gromov-Hausdorff distance}

A map $f: X \rightarrow Y$ between metric spaces, not necessarily continuous, is called an $\varepsilon$-approximation if an $\varepsilon$-neighborhood of its image covers all of $Y$ and $\mid d_{X}(p, q)-$ $d_{Y}(f(p), f(q)) \mid \leq \varepsilon$ for all $p, q \in X$. The Gromov-Hausdorff distance between two compact metric spaces $X$ and $Y$ is the infimum of $\varepsilon>0$ such that there exist $\varepsilon$-approximations 
$X \rightarrow Y$ and $Y \rightarrow X$. This distance function between (isometry classes of) compact metric spaces and the corresponding notion of convergence were pioneered by Gromov [19].

Gromov-Hausdorff convergence can be easily extended to pointed complete metric spaces, by declaring that $\left(X_{i}, p_{i}\right)$ converges to $(X, p)$ if, for all $r>0$, the ball of radius $r$ in $X_{i}$ centered at $p_{i}$ Gromov-Hausdorff converges to the ball of radius $r$ in $X$ centered at $p$. Furthermore, an equivariant extension of this notion was introduced by Fukaya [15] and achieved its final form in Fukaya and Yamaguchi [16].

\subsection{Converging sequences of flat manifolds}

The case of flat tori is essentially due to Mahler [27], see also Pansu [29, Cor., p. 70]. For the convenience of the reader, we provide an alternative proof based on a general construction of limits of isometric group actions due to Fukaya and Yamaguchi [16].

Proposition 3.1 The Gromov-Hausdorff limit of a sequence $\left\{\left(T^{n}, g_{i}\right)\right\}_{i \in \mathbb{N}}$ of flat tori with bounded diameter is a flat torus $\left(T^{m}, h\right)$ of dimension $0 \leq m \leq n$.

Proof Let $\left\{\left(X_{i}, p_{i}\right)\right\}_{i \in \mathbb{N}}$ be a sequence of pointed complete Riemannian manifolds (more generally, pointed locally compact length spaces) that Gromov-Hausdorff converges to a limit space $(X, p)$ and $G_{i} \subset \operatorname{Iso}\left(X_{i}\right)$ be closed subgroups of isometries. By Fukaya and Yamaguchi [16, Prop. 3.6], there is a closed subgroup $G \subset \operatorname{Iso}(X)$ such that $\left\{\left(X_{i}, G_{i}, p_{i}\right)\right\}_{i \in \mathbb{N}}$ converges in equivariant Gromov-Hausdorff sense to $(X, G, p)$. In particular, the orbit spaces $\left\{\left(X_{i} / G_{i},\left[p_{i}\right]\right)\right\}_{i \in \mathbb{N}}$ Gromov-Hausdorff converge to the orbit space $(X / G,[p])$.

Each flat torus $\left(T^{n}, g_{i}\right)$ is isometric to $\mathbb{R}^{n} / G_{i}$, where $G_{i} \subset \operatorname{Iso}\left(\mathbb{R}^{n}\right)$ is a lattice, that is, a discrete subgroup consisting only of translations. Applying the aforementioned result to the constant sequence $\left(X_{i}, p_{i}\right)=\left(\mathbb{R}^{n}, 0\right)$ and the lattices $G_{i}$, it follows that there exists a closed subgroup $G \subset \operatorname{Iso}\left(\mathbb{R}^{n}\right)$ such that $\left(T^{n}, g_{i}\right)$ converge in Gromov-Hausdorff sense to $\mathbb{R}^{n} / G$. We claim that this limit group $G \subset \operatorname{Iso}\left(\mathbb{R}^{n}\right)$ is a degenerate lattice, that is, $G \cong L \times \mathbb{R}^{n-m}$ for some $0 \leq m \leq n$, where $L \subset \operatorname{Iso}\left(\mathbb{R}^{m}\right)$ is a lattice; hence, $\mathbb{R}^{n} / G$ is isometric to the flat torus $T^{m}=\mathbb{R}^{m} / L$.

To prove this claim, which concludes the proof of the proposition, we use that metric properties of the isometries $G_{i}$ are preserved in the limit $G$ by its inductive-projective construction. Translations are metrically characterized as isometries that have constant displacement, i.e., isometries that move all points in $\mathbb{R}^{n}$ by the same distance. Since all elements of $G_{i}$ satisfy this property, for all $i \in \mathbb{N}$, also all elements of $G$ satisfy it and are hence translations. Moreover, the only closed subgroups $G$ of $\operatorname{Iso}\left(\mathbb{R}^{n}\right)$ that consist of translations are degenerate lattices in subspaces of $\mathbb{R}^{n}$. Indeed, the identity connected component $G_{0} \cong \mathbb{R}^{n-m}$ is a subspace of $\mathbb{R}^{n}$ and the quotient $L=G / \mathbb{R}^{n-m}$ is discrete and abelian, hence a lattice in a subspace $V \subset \mathbb{R}^{n}$ with $V \cap \mathbb{R}^{n-m}=\{0\}$. As the limit space $\mathbb{R}^{n} / G$ is compact, since $\mathbb{R}^{n} / G_{i}$ have bounded diameter, we have that $V \cong \mathbb{R}^{m}$ is a complement of $G_{0} \cong \mathbb{R}^{n-m}$. This proves that $G \cong L \times \mathbb{R}^{n-m}$ is a degenerate lattice in $\mathbb{R}^{n}$.

By the Bieberbach Theorems, there are only finitely many diffeomorphism types of closed flat $n$-manifolds for any given $n \in \mathbb{N}$. Thus, up to subsequences, we may assume that any Gromov-Hausdorff converging sequence of closed flat $n$-manifolds is of the form $\left\{\left(M, g_{i}\right)\right\}_{i \in \mathbb{N}}$, where $g_{i}$ are flat metrics on a fixed manifold $M$.

Proposition 3.2 Let $\left\{\left(M, g_{i}\right)\right\}_{i \in \mathbb{N}}$ be a Gromov-Hausdorff sequence of closed flat $n$ manifolds that converges to a limit metric space $\left(X, d_{X}\right)$. Then $\left(X, d_{X}\right)$ is isometric to a flat orbifold $T^{m} / H$, where $0 \leq m \leq n$ and $H \subset \mathrm{O}(n)$ is a finite subgroup conjugate to the holonomy group of $\left(M, g_{i}\right)$ that acts isometrically on $T^{m}$. 
Proof Without loss of generality, we may assume that the holonomy groups of $\left(M, g_{i}\right)$ are all equal to $H \subset \mathrm{O}(n)$, see Corollary 4.10. Let $\left(T^{n}, \tilde{g}_{i}\right) \rightarrow\left(M, g_{i}\right)$ be the Riemannian coverings by flat tori whose group of deck transformations is $H$. Then,

$$
\frac{1}{|H|} \operatorname{diam}\left(T^{n}, \tilde{g}_{i}\right) \leq \operatorname{diam}\left(M, g_{i}\right) \leq \operatorname{diam}\left(T^{n}, \tilde{g}_{i}\right) \text {. }
$$

Therefore, $\operatorname{diam}\left(T^{n}, \widetilde{g}_{i}\right) \leq 2|H| \operatorname{diam}\left(X, d_{X}\right)$ and, by Gromov's Compactness Theorem and Proposition 3.1, there is a subsequence of $\left\{\left(T^{n}, \widetilde{g}_{i}\right)\right\}_{i \in \mathbb{N}}$ that Gromov-Hausdorff converges to a flat torus $\left(T^{m}, h\right), 0 \leq m \leq n$. Up to passing to a new subsequence, this convergence is in equivariant Gromov-Hausdorff sense with respect to the isometric $H$-actions, by Fukaya and Yamaguchi [16, Prop. 3.6]. Thus, the orbit spaces $\left\{\left(T^{n}, \widetilde{g}_{i}\right) / H\right\}_{i \in \mathbb{N}}$, which are isometric to $\left\{\left(M, g_{i}\right)\right\}_{i \in \mathbb{N}}$, Gromov-Hausdorff converge to the orbit space $\left(T^{m}, h\right) / H$.

To finish the proof of Theorem A, it only remains to prove the following:

Proposition 3.3 Any flat orbifold is the Gromov-Hausdorfflimit of a sequence of closed flat manifolds.

Proof Let $\mathcal{O}=\mathbb{R}^{n} / \pi_{\mathcal{O}}$ be a flat orbifold, with orbifold fundamental group given by the crystallographic group $\pi_{\mathcal{O}} \subset \operatorname{Iso}\left(\mathbb{R}^{n}\right)$. By Theorem 2.3 (of Auslander and Kuranishi [2]), there exists a closed flat manifold $M=\mathbb{R}^{m} / \pi_{M}$ whose fundamental group $\pi_{M} \subset \operatorname{Iso}\left(\mathbb{R}^{m}\right)$ is a Bieberbach group with the same holonomy, that is, $H_{\pi_{M}} \cong H_{\pi_{\mathcal{O}}}$. Denote this finite group by $H$, and consider its (isometric) actions on the flat tori $T^{n}=\mathbb{R}^{n} / L_{\pi_{\mathcal{O}}}$ and $T^{m}=\mathbb{R}^{m} / L_{\pi_{M}}$. Clearly, the orbit spaces of these actions are $T^{n} / H=\mathcal{O}$ and $T^{m} / H=M$. Since the $H$-action on $T^{m}$ is free, so is the diagonal $H$-action on the product $T^{n} \times T^{m}$. Thus, $N=\left(T^{n} \times T^{m}\right) / H$ is a closed flat manifold. The product metrics $g_{\lambda}=g_{T^{n}} \oplus \lambda g_{T^{m}}, \lambda>0$, are invariant under the $H$-action and hence descend to flat metrics on $N$. The closed flat manifolds $\left\{\left(N, g_{\lambda}\right)\right\}_{\lambda>0}$ clearly Gromov-Hausdorff converge to $\mathcal{O}$ as $\lambda \searrow 0$.

\section{Teichmüller space and moduli space of flat metrics}

In this section, we study the Teichmüller space and moduli space of flat metrics on a closed flat orbifold (or manifold) and prove Theorem B in the Introduction.

\subsection{Teichmüller space}

It follows from the Bieberbach Theorems that any flat metrics on a given closed flat orbifold (or manifold) $\mathcal{O}=\mathbb{R}^{n} / \pi$ are of the form $g_{\pi^{\prime}}$ where $\pi^{\prime}=(A, v) \pi(A, v)^{-1}$ for some $(A, v) \in \operatorname{Aff}\left(\mathbb{R}^{n}\right)$. In this situation,

$$
H_{\pi^{\prime}}=A H_{\pi} A^{-1} \text { and } L_{\pi^{\prime}}=A\left(L_{\pi}\right) .
$$

Distinguishing isometry classes of such metrics is straightforward (see also [4]):

Lemma 4.1 The metrics $g_{\pi}$ and $g_{\pi^{\prime}}$ are isometric, where $\pi^{\prime}=(A, v) \pi(A, v)^{-1}$, if and only if $A=B C$, where $B \in \mathrm{O}(n)$ and $C \in \mathcal{N}_{\pi}:=\mathfrak{r}\left(N_{\operatorname{Aff}\left(\mathbb{R}^{n}\right)}(\pi)\right)$.

Proof By lifting isometries to $\mathbb{R}^{n}$, it is clear that $g_{\pi}$ and $g_{\pi^{\prime}}$ are isometric if and only if there is $(B, w) \in \operatorname{Iso}\left(\mathbb{R}^{n}\right)$ such that $(B, w) \pi(B, w)^{-1}=(A, v) \pi(A, v)^{-1}$, that is, $(C, z):=(B, w)^{-1}(A, v) \in N_{\mathrm{Aff}\left(\mathbb{R}^{n}\right)}(\pi)$. If $g_{\pi}$ and $g_{\pi^{\prime}}$ are isometric, then clearly $A=B C$, 
with $B \in \mathrm{O}(n)$ and $C \in \mathcal{N}_{\pi}$. Conversely, if $A=B C$, with $B \in \mathrm{O}(n)$ and $C \in \mathcal{N}_{\pi}$, there is $z \in \mathbb{R}^{n}$ such that $(C, z) \in N_{\mathrm{Aff}\left(\mathbb{R}^{n}\right)}(\pi)$. Set $w=v-B z$, so that $(A, v)=(B, w)(C, z)$. Clearly, $(B, w)^{-1}(A, v) \in N_{\mathrm{Aff}\left(\mathbb{R}^{n}\right)}(\pi)$, so $g_{\pi}$ and $g_{\pi^{\prime}}$ are isometric.

While Lemma 4.1 provides the appropriate equivalence relation to distinguish isometry classes, it remains to characterize the space where these relations take place. For a given crystallographic group $\pi \subset \operatorname{Iso}\left(\mathbb{R}^{n}\right)$, we let

$$
\mathcal{C}_{\pi}:=\left\{A \in \mathrm{GL}(n): A H_{\pi} A^{-1} \subset \mathrm{O}(n)\right\} .
$$

It is easy to see that $A H_{\pi} A^{-1} \subset \mathrm{O}(n)$ is equivalent to $A^{\mathrm{t}} A \in Z_{\mathrm{GL}(n)}\left(H_{\pi}\right)$, where $A^{\mathrm{t}}$ is the transpose of $A$. The set $\mathcal{C}_{\pi}$ is a closed cone in $\mathrm{GL}(n)$ that contains $N_{\mathrm{GL}(n)}\left(H_{\pi}\right)$. There are natural actions on $\mathcal{C}_{\pi}$ by matrix multiplication, on the left by $\mathrm{O}(n)$ and on the right by $N_{\mathrm{GL}(n)}\left(H_{\pi}\right)$. In this context, it is natural to introduce the following:

Definition 4.2 The Teichmüller space $\mathcal{T}_{\text {flat }}(\mathcal{O})$ of the flat orbifold $\mathcal{O}=\mathbb{R}^{n} / \pi$ is the orbit space $\mathrm{O}(n) \backslash \mathcal{C}_{\pi}$ of the left $\mathrm{O}(n)$-action on $\mathcal{C}_{\pi}$.

The Teichmüller space $\mathcal{T}_{\text {flat }}(\mathcal{O})$ is a real analytic manifold diffeomorphic to $\mathbb{R}^{d}$ and is described further in Sect. 4.4. It can also be obtained as the deformation space of certain $(X, G)$-structures, see Sect. 4.3.

\subsection{Moduli space}

The moduli space $\mathcal{M}_{\text {flat }}(\mathcal{O})$ of the flat orbifold $\mathcal{O}=\mathbb{R}^{n} / \pi$ is defined as the set of isometry classes of flat metrics on $\mathcal{O}$. Considering the restriction to $\mathcal{N}_{\pi}$ of the right $N_{\mathrm{GL}(n)}\left(H_{\pi}\right)$-action on $\mathcal{C}_{\pi}$, the following identification follows directly from Lemma 4.1, cf. Wolf [34, Thm. 1].

Proposition 4.3 $\mathcal{M}_{\text {flat }}(\mathcal{O})=\mathcal{T}_{\text {flat }}(\mathcal{O}) / \mathcal{N}_{\pi}$.

Note that the right $\mathcal{N}_{\pi}$-action on $\mathcal{C}_{\pi}$ need not be free, so $\mathcal{M}_{\text {flat }}(\mathcal{O})$ may have (isolated) singularities; this may also be the case if $\mathcal{O}$ is a smooth manifold. As indicated by Proposition 4.3, the group $\mathcal{N}_{\pi}$ is related to the mapping class group in this Teichmüller theory (see Remark 4.7) and moreover satisfies the following:

Proposition 4.4 $\mathcal{N}_{\pi}$ is isomorphic to a discrete subgroup of the group $\operatorname{Aff}(\mathcal{O}) \cong$ $N_{\operatorname{Aff}\left(\mathbb{R}^{n}\right)}(\pi) / Z_{\operatorname{Aff}\left(\mathbb{R}^{n}\right)}(\pi)$ of affine diffeomorphisms of $\mathcal{O}=\mathbb{R}^{n} / \pi$.

Proof It is easy to see that $\mathcal{N}_{\pi}$ is a countable (Lie) group and thus discrete. In fact, if $\pi$ is a lattice, then $\mathcal{N}_{\pi}$ is a conjugate of $\mathrm{GL}(n, \mathbb{Z})$ inside $\mathrm{GL}(n)$ and hence countable; while for a general crystallographic group $\pi$, one has $\mathcal{N}_{\pi} \subset \mathcal{N}_{L_{\pi}}$.

A diffeomorphism $\phi: \mathcal{O} \rightarrow \mathcal{O}$ that preserves the affine structure of the flat orbifold $\mathcal{O}=\mathbb{R}^{n} / \pi$ endowed with the metric $g_{\pi}$ lifts to an affine diffeomorphism $\widetilde{\phi}: \mathbb{R}^{n} \rightarrow \mathbb{R}^{n}$. Conversely, an affine diffeomorphism $\widetilde{\phi}$ of $\mathbb{R}^{n}$ descends to an affine diffeomorphism $\phi$ of $\left(\mathcal{O}, g_{\pi}\right)$ if and only if $\widetilde{\phi}$ normalizes $\pi$. We thus have a surjective homomorphism from the normalizer of $\pi$ in $\operatorname{Aff}\left(\mathbb{R}^{n}\right)$ to the group $\operatorname{Aff}(\mathcal{O})$ of affine diffeomorphisms of $\left(\mathcal{O}, g_{\pi}\right)$, given by $N_{\operatorname{Aff}\left(\mathbb{R}^{n}\right)}(\pi) \ni \widetilde{\phi} \mapsto \phi \in \operatorname{Aff}(\mathcal{O})$, whose kernel is the centralizer $Z_{\operatorname{Aff}\left(\mathbb{R}^{n}\right)}(\pi)$. This establishes the isomorphism:

$$
\operatorname{Aff}(\mathcal{O}) \cong N_{\text {Aff }\left(\mathbb{R}^{n}\right)}(\pi) / Z_{\text {Aff }\left(\mathbb{R}^{n}\right)}(\pi) .
$$

Note that the group of affine diffeomorphisms of the closed flat orbifold $\mathcal{O}=\mathbb{R}^{n} / \pi$ does not depend on the flat metric, but only on the isomorphism class of the crystallographic group $\pi$, by the Bieberbach Theorems. 
The group $\mathcal{N}_{\pi}=\mathfrak{r}\left(N_{\mathrm{Aff}\left(\mathbb{R}^{n}\right)}(\pi)\right)$ is isomorphic to the quotient of $N_{\mathrm{Aff}\left(\mathbb{R}^{n}\right)}(\pi)$ by the kernel of the projection homomorphism $\mathfrak{r}: N_{\mathrm{Aff}\left(\mathbb{R}^{n}\right)}(\pi) \rightarrow N_{\mathrm{GL}(n)}\left(H_{\pi}\right)$, which is given by $N_{\mathrm{Aff}\left(\mathbb{R}^{n}\right)}(\pi) \cap\left(\{\mathrm{Id}\} \times \mathbb{R}^{n}\right)=\left\{(\mathrm{Id}, w): A w-w \in L_{\pi}\right.$, for all $\left.A \in H_{\pi}\right\}$. Clearly, the latter contains $Z_{\mathrm{Aff}\left(\mathbb{R}^{n}\right)}(\pi)=\left\{(\mathrm{Id}, w): A w=w\right.$, for all $\left.A \in H_{\pi}\right\}$, and hence, $\mathcal{N}_{\pi}$ is isomorphic to a subgroup of $\operatorname{Aff}(\mathcal{O})$, concluding the proof.

Remark 4.5 In general, $\mathcal{N}_{\pi}$ is a proper subgroup of $\operatorname{Aff}_{\text {flat }}(\mathcal{O})$. For instance, if $\mathbb{R}^{2} / \pi$ is the Klein bottle, then $L_{\pi}=\mathbb{Z}^{2}$, and the orthogonal representation of $H_{\pi} \cong \mathbb{Z}_{2}$ on $\mathbb{R}^{2}$ is by reflection about the $x$-axis, so $Z_{\mathrm{Aff}\left(\mathbb{R}^{2}\right)}(\pi)=\left\{(\operatorname{Id}, w): w=\left(w_{1}, 0\right) \in \mathbb{Z}^{2}\right\}$, while $N_{\text {Aff }\left(\mathbb{R}^{2}\right)}(\pi) \cap\left(\{\operatorname{Id}\} \times \mathbb{R}^{2}\right)=\left\{(\operatorname{Id}, w): w=\left(w_{1}, w_{2}\right) \in \mathbb{Z}^{2}\right\}$.

\subsection{Deformations of $(X, G)$-structures}

The Teichmüller space $\mathcal{T}_{\text {flat }}(M)$ and the moduli space $\mathcal{M}_{\text {flat }}(M)$ of a flat manifold can also be described using the language of $(X, G)$-structures [33]. Given a Lie group $G$ and a $G$ homogeneous space $X$, an $(X, G)$-structure on a manifold $M$ is a (maximal) atlas of charts on $M$ with values in $X$, whose transition maps are given by restrictions of elements of $G$. Setting $X=\mathbb{R}^{n}$ and $G=\operatorname{Iso}\left(\mathbb{R}^{n}\right)$, an $(X, G)$-structure on a manifold $M$ is precisely a flat Riemannian metric on $M$.

A general deformation theory of $(X, G)$-structures on a given manifold $M$ is discussed in $[3,18,33]$. Using the action of the diffeomorphism group $\operatorname{Diff}(M)$ on the space of $(X, G)$ structures on $M$, one defines the corresponding deformation space and the moduli space, respectively, as

$$
\begin{aligned}
& \mathcal{D}(M)=\{(X, G) \text {-structures on } M\} / \operatorname{Diff}_{0}(M), \\
& \mathcal{M}(M)=\{(X, G) \text {-structures on } M\} / \operatorname{Diff}(M),
\end{aligned}
$$

where $\operatorname{Diff}_{0}(M) \subset \operatorname{Diff}(M)$ is the connected component of the identity. The mapping class group of $M$ is defined as $\operatorname{MCG}(M)=\operatorname{Diff}(M) / \operatorname{Diff}_{0}(M)$, so that $\mathcal{M}(M)=$ $\mathcal{D}(M) / \operatorname{MCG}(M)$.

Proposition 4.6 For $(X, G)=\left(\mathbb{R}^{n}\right.$, Iso $\left.\left(\mathbb{R}^{n}\right)\right)$, the deformation space $\mathcal{D}(M)$ and the moduli space $\mathcal{M}(M)$ can be, respectively, identified with the Teichmüller space $\mathcal{T}_{\text {flat }}(M)$ and the moduli space $\mathcal{M}_{\text {flat }}(M)$.

Proof Assume $M=\mathbb{R}^{n} / \pi$, where $\pi \subset \operatorname{Iso}\left(\mathbb{R}^{n}\right)$ is a Bieberbach group, and consider the set $\operatorname{Inj}\left(\pi, \operatorname{Iso}\left(\mathbb{R}^{n}\right)\right)$ of injective homomorphisms of $\pi$ into Iso $\left(\mathbb{R}^{n}\right)$. There is a left action of $\operatorname{Iso}\left(\mathbb{R}^{n}\right)$ on $\operatorname{Inj}\left(\pi, \operatorname{Iso}\left(\mathbb{R}^{n}\right)\right)$ by conjugation, i.e., composition with inner automorphisms. The deformation space $\mathcal{D}(M)$ is identified with the quotient $\operatorname{Iso}\left(\mathbb{R}^{n}\right) \backslash \operatorname{Inj}\left(\pi, \operatorname{Iso}\left(\mathbb{R}^{n}\right)\right)$, see [3, Prop. 1.6]. By the Bieberbach Theorems, $\operatorname{Inj}\left(\pi, \operatorname{Iso}\left(\mathbb{R}^{n}\right)\right)$ can be identified with $\mathcal{C}_{\pi} \times \mathbb{R}^{n}$. Using this identification, the action of $\operatorname{Iso}\left(\mathbb{R}^{n}\right)=\mathrm{O}(n) \ltimes \mathbb{R}^{n}$ on $\operatorname{Inj}\left(\pi, \operatorname{Iso}\left(\mathbb{R}^{n}\right)\right)$ is given by left multiplication; hence, the quotient $\mathcal{D}(M)=\operatorname{Iso}\left(\mathbb{R}^{n}\right) \backslash \operatorname{Inj}\left(\pi, \operatorname{Iso}\left(\mathbb{R}^{n}\right)\right)$ is identified with the quotient $\mathcal{T}_{\text {flat }}(M)=\mathrm{O}(n) \backslash \mathcal{C}_{\pi}$, cf. Definition 4.2.

By a generalization of the Dehn-Nielsen-Baer Theorem to flat manifolds, the action of $\operatorname{Diff}(M)$ on the loop space of $M$ induces an isomorphism from $\operatorname{MCG}(M)$ to the group $\operatorname{Out}(\pi)$ of outer automorphisms of $\pi$. Moreover, $\operatorname{Out}(\pi)$ is isomorphic to $\operatorname{Aff}(M) / \operatorname{Aff}_{0}(M)$, see [10, Thm. 6.1]. Using [10, Lemma 6.1], it follows that the orbits of the action of this group on $\operatorname{Iso}\left(\mathbb{R}^{n}\right) \backslash \operatorname{Inj}\left(\pi, \operatorname{Iso}\left(\mathbb{R}^{n}\right)\right)$ are the same as the orbits of $N_{\operatorname{Aff}\left(\mathbb{R}^{n}\right)}(\pi)$ acting by composition on the right with conjugations. Under the identification of $\mathcal{D}(M)=\operatorname{Iso}\left(\mathbb{R}^{n}\right) \backslash \operatorname{Inj}\left(\pi, \operatorname{Iso}\left(\mathbb{R}^{n}\right)\right)$ with $\mathcal{T}_{\text {flat }}(M)=\mathrm{O}(n) \backslash \mathcal{C}_{\pi}$, the action of $N_{\text {Aff }\left(\mathbb{R}^{n}\right)}(\pi)$ coincides with the action of the group 
$\mathcal{N}_{\pi}$ (see Lemma 4.1) by right multiplication. Thus, $\mathcal{M}(M)$ is identified with $\mathcal{M}_{\text {flat }}(M)$, cf. Proposition 4.3 .

Remark 4.7 By the results quoted above, given a flat manifold $M=\mathbb{R}^{n} / \pi$, there are isomorphisms

$$
\operatorname{Diff}(M) / \operatorname{Diff}_{0}(M)=\operatorname{MCG}(M) \cong \operatorname{Out}(\pi) \cong \operatorname{Aff}(M) / \operatorname{Aff}_{0}(M) .
$$

\subsection{Algebraic description of Teichmüller space}

Let $\mathcal{O}=\mathbb{R}^{n} / \pi$ be a closed flat orbifold and $\mathcal{T}_{\text {flat }}(\mathcal{O})$ be its Teichmüller space. We now employ simple algebraic considerations to identify $\mathcal{T}_{\text {flat }}(\mathcal{O})$ with a product of (noncompact) homogeneous spaces, proving Theorem B.

Proposition 4.8 The cone $\mathcal{C}_{\pi}$ defined in (4) satisfies $\mathcal{C}_{\pi}=\mathrm{O}(n) \cdot Z_{\mathrm{GL}(n)}\left(H_{\pi}\right)$.

Proof Clearly, $\mathrm{O}(n) \cdot Z_{\mathrm{GL}(n)}\left(H_{\pi}\right) \subset \mathcal{C}_{\pi}$. Choose $A \in \mathcal{C}_{\pi}$, and write the polar decomposition $A=O P$, with $P=\left(A^{\mathrm{t}} A\right)^{\frac{1}{2}}$ and $O \in \mathrm{O}(n)$. Since $A^{\mathrm{t}} A$ centralizes $H_{\pi}$, also $P$ centralizes $H_{\pi}$. Thus, $A \in \mathrm{O}(n) \cdot Z_{\mathrm{GL}(n)}\left(H_{\pi}\right)$, concluding the proof.

The following is an immediate consequence of Proposition 4.8 and Definition 4.2.

Corollary 4.9 The Teichmüller space $\mathcal{T}_{\text {flat }}(\mathcal{O})$ is identified with the quotient space $Z_{\mathrm{GL}(n)}\left(H_{\pi}\right) / \simeq$, where $A \simeq B$ if there exists $O \in \mathrm{O}(n)$ with $B=O \cdot A$. Thus, it can be described as the space of right cosets $\left(Z_{\mathrm{GL}(n)}\left(H_{\pi}\right) \cap \mathrm{O}(n)\right) \backslash Z_{\mathrm{GL}(n)}\left(H_{\pi}\right)$.

According to (3), different flat metrics on the same closed orbifold have conjugate holonomy groups. A more precise statement follows from Proposition 4.8:

Corollary 4.10 Any flat metric $g$ on $\mathcal{O}=\mathbb{R}^{n} / \pi$ is isometric to a (flat) metric on $\mathcal{O}$ that has the same holonomy as $g_{\pi}$.

Proof There is a crystallographic group $\pi^{\prime} \subset \operatorname{Iso}\left(\mathbb{R}^{n}\right)$ such that $g=g_{\pi^{\prime}}$. By Proposition 4.8, there exist $O \in \mathrm{O}(n)$ and $A \in Z_{\mathrm{GL}(n)}\left(H_{\pi}\right)$ such that $\pi^{\prime}=O A \cdot \pi \cdot A^{-1} O^{\mathrm{t}}$. Thus, $g_{\pi^{\prime}}$ is isometric to $g_{\pi^{\prime \prime}}$, where $\pi^{\prime \prime}=A \cdot \pi \cdot A^{-1}$, and $H_{\pi^{\prime \prime}}=H_{\pi}$ since $A$ normalizes $H_{\pi}$.

In order to achieve a more precise description of $Z_{\mathrm{GL}(n)}\left(H_{\pi}\right)$, leading to the proof of Theorem B via Corollary 4.9, we use the decomposition of $\mathbb{R}^{n}$ into $H_{\pi}$-isotypic components. By a result of Hiss and Szczepański [22], see Theorem 2.4, there are always nontrivial invariant subspaces of the orthogonal $H_{\pi}$-representation on $\mathbb{R}^{n}$. Recall that a nonzero invariant subspace $V$ is irreducible if it contains no proper invariant subspaces, or, equivalently, if every nonzero element of the vector space $\operatorname{End}_{H_{\pi}}(V)$ of linear equivariant endomorphisms of $V$ is an isomorphism. In this situation, $\operatorname{End}_{H_{\pi}}(V)$ is an associative real division algebra and hence isomorphic to one of $\mathbb{R}, \mathbb{C}$, or $\mathbb{H}$. The irreducible $V$ is called of real, complex, or quaternionic type, according to the isomorphism type of $\operatorname{End}_{H_{\pi}}(V)$.

Consider the decomposition of the orthogonal $H_{\pi}$-representation into irreducibles,

$$
\mathbb{R}^{n}=\underbrace{V_{1,1} \oplus \cdots \oplus V_{1, m_{1}}}_{W_{1}} \oplus \cdots \oplus \underbrace{V_{l, 1} \oplus \cdots \oplus V_{l, m_{l}}}_{W_{l}},
$$

where each $V_{i, j}$ is irreducible and $V_{i, j}$ is isomorphic to $V_{i^{\prime}, j^{\prime}}$ if and only if $i=i^{\prime}$, so that $W_{i}=\bigoplus_{j=1}^{m_{i}} V_{i, j}, i=1, \ldots, l$ are the so-called isotypic components. Let $\mathbb{K}_{i}$ be the real 
division algebra $\mathbb{R}, \mathbb{C}$, or $\mathbb{H}$, according to $W_{i}$ consisting of irreducibles $V_{i, j}$ of real, complex, or quaternionic type.

$\operatorname{As} Z_{\mathrm{GL}(n)}\left(H_{\pi}\right)=\operatorname{End}_{H_{\pi}}\left(\mathbb{R}^{n}\right) \cap \mathrm{GL}(n)$, and $\operatorname{End}_{H_{\pi}}\left(\mathbb{R}^{n}\right) \cong \prod_{i=1}^{l} \operatorname{End}_{H_{\pi}}\left(W_{i}\right)$ by Schur's Lemma, it follows that there is an isomorphism:

$$
Z_{\mathrm{GL}(n)}\left(H_{\pi}\right) \cong \prod_{i=1}^{l} \mathrm{GL}\left(m_{i}, \mathbb{K}_{i}\right) .
$$

For each isotypic component $W_{i}$, it is clear that $\operatorname{End}_{H_{\pi}}\left(W_{i}\right) \cap \mathrm{O}\left(W_{i}\right) \cong \mathrm{O}\left(m_{i}, \mathbb{K}_{i}\right)$, where

$$
\mathrm{O}(m, \mathbb{K}):= \begin{cases}\mathrm{O}(m), & \text { if } \quad \mathbb{K}=\mathbb{R} ; \\ \mathrm{U}(m), & \text { if } \mathbb{K}=\mathbb{C} ; \\ \operatorname{Sp}(m), & \text { if } \mathbb{K}=\mathbb{H} .\end{cases}
$$

Thus, from Corollary 4.9 and these isomorphisms,

$$
\mathcal{T}_{\text {flat }}(\mathcal{O}) \cong \frac{Z_{\mathrm{GL}(n)}\left(H_{\pi}\right)}{Z_{\mathrm{GL}(n)}\left(H_{\pi}\right) \cap \mathrm{O}(n)} \cong \frac{\prod_{i=1}^{l} \operatorname{End}_{H_{\pi}}\left(W_{i}\right) \cap \mathrm{GL}\left(W_{i}\right)}{\prod_{i=1}^{l} \operatorname{End}_{H_{\pi}}\left(W_{i}\right) \cap \mathrm{O}\left(W_{i}\right)} \cong \prod_{i=1}^{l} \frac{\mathrm{GL}\left(m_{i}, \mathbb{K}_{i}\right)}{\mathrm{O}\left(m_{i}, \mathbb{K}_{i}\right)},
$$

which concludes the proof of Theorem B in the Introduction.

\section{Examples of Teichmüller spaces}

In this section, we apply Theorem B to compute the Teichmüller space of some flat manifolds and orbifolds.

\subsection{Flat tori}

If $\pi=L_{\pi}$ is a lattice, then $H_{\pi}$ is trivial and $M=\mathbb{R}^{n} / \pi$ is a flat torus $T^{n}$. In this case, Theorem B gives:

$$
\mathcal{T}_{\text {flat }}\left(T^{n}\right) \cong \frac{\mathrm{GL}(n)}{\mathrm{O}(n)} \cong \mathbb{R}^{n(n+1) / 2} .
$$

Furthermore, it is easy to see that $N_{\mathrm{Aff}\left(\mathbb{R}^{n}\right)}(\pi)=\mathbb{R}^{n} \ltimes \mathrm{GL}(n, \mathbb{Z})$, so $\mathcal{N}_{\pi} \cong \operatorname{GL}(n, \mathbb{Z})$ is the discrete subgroup of integer matrices with determinant \pm 1 . Thus, $\mathcal{M}_{\text {flat }}\left(T^{n}\right)=$ $\mathrm{O}(n) \backslash \mathrm{GL}(n) / \mathrm{GL}(n, \mathbb{Z})$, cf. Wolf [34, Cor].

Remark 5.1 Flat metrics on the 2-torus $T^{2}$ are often parametrized using the upper half plane, identifying each flat metric $g_{\pi}$ with $w / z \in \mathbb{C}$, where $z, w \in \mathbb{C}$ are chosen so that $\pi=$ $\operatorname{span}_{\mathbb{Z}}\{z, w\}, z \in \mathbb{R}, z>0$, and $\operatorname{Im} w>0$. This parametrization clearly identifies homothetic metrics, while they are distinct in $\mathcal{T}_{\text {flat }}\left(T^{2}\right)$ and $\mathcal{M}_{\text {flat }}\left(T^{2}\right)$.

The moduli space $\mathcal{M}_{\text {flat }}\left(T^{2}\right)=\mathcal{T}_{\text {flat }}\left(T^{2}\right) / \mathrm{GL}(2, \mathbb{Z})$ has two singular strata of dimension 1 , corresponding to hexagonal and square lattices. Furthermore, it has one end whose boundary at infinity is a ray $[0,+\infty)$, corresponding to the lengths of circles to which $T^{2}$ can collapse. For details, see $[14, \S 12.1]$.

\subsection{Cyclic holonomy}

Assume that $H_{\pi} \subset \mathrm{O}(n)$ is a cyclic group, and choose a generator $A \in H_{\pi}$. Up to rewriting $A$ in its real canonical form, we may assume that it is block diagonal, with $1 \times 1$ and $2 \times 2$ 
blocks, where $m_{1}$ diagonal entries are $1, m_{2}$ diagonal entries are -1 , and each of the $2 \times 2$ blocks is a rotation matrix

$$
\left(\begin{array}{rr}
\cos \theta_{i} & -\sin \theta_{i} \\
\sin \theta_{i} & \cos \theta_{i}
\end{array}\right), \quad \theta_{i} \in(0, \pi), i=3, \ldots, l,
$$

appearing $m_{i}$ times. Therefore, the decomposition (6) has 2 isotypic components of real type with dimensions $m_{1}$ and $m_{2}$, namely $W_{1}=\operatorname{ker}(A-\mathrm{Id}) \neq\{0\}$, and $W_{2}=\operatorname{ker}(A+\mathrm{Id})$, and all other isotypic components $W_{i}, i=3, \ldots, l$, are of complex type and consist of $m_{i}$ copies of $\mathbb{R}^{2}$.

The centralizer $Z_{\mathrm{GL}(n)}\left(H_{\pi}\right)$ consists of block diagonal matrices whose first two blocks (corresponding to $W_{1}$ and $W_{2}$ ) are any invertible $m_{1} \times m_{1}$ and $m_{2} \times m_{2}$ matrices, while the remaining blocks are $m_{i} \times m_{i}$ invertible matrices which are also complex linear. In other words, there is an isomorphism

$$
Z_{\mathrm{GL}(n)}\left(H_{\pi}\right) \cong \mathrm{GL}\left(m_{1}, \mathbb{R}\right) \times \mathrm{GL}\left(m_{2}, \mathbb{R}\right) \times \prod_{i=3}^{l} \mathrm{GL}\left(m_{i}, \mathbb{C}\right) .
$$

Therefore, according to Theorem $\mathrm{B}$, the Teichmüller space of $M=\mathbb{R}^{n} / \pi$ is:

$$
\mathcal{T}_{\text {flat }}(M) \cong \frac{\mathrm{GL}\left(m_{1}, \mathbb{R}\right)}{\mathrm{O}\left(m_{1}\right)} \times \frac{\mathrm{GL}\left(m_{2}, \mathbb{R}\right)}{\mathrm{O}\left(m_{2}\right)} \times \prod_{i=3}^{l} \frac{\mathrm{GL}\left(m_{i}, \mathbb{C}\right)}{\mathrm{U}\left(m_{i}\right)} \cong \mathbb{R}^{d},
$$

where $d=\frac{1}{2} m_{1}\left(m_{1}+1\right)+\frac{1}{2} m_{2}\left(m_{2}+1\right)+\sum_{i=3}^{l} m_{i}^{2}$.

The case in which $H_{\pi} \cong \mathbb{Z}_{p}$, with $p$ prime, is particularly interesting [10, § IV.7]. If $p=2$, then all $H_{\pi}$-isotypic components are of real type and hence $\mathcal{T}_{\text {flat }}(M) \cong \mathbb{R}^{d}$, with $d=\frac{1}{2} m_{1}\left(m_{1}+1\right)+\frac{1}{2} m_{2}\left(m_{2}+1\right)$. For instance, if $M=\mathbb{R}^{2} / \pi$ is the Klein bottle, then $m_{1}=m_{2}=1$ and $\mathcal{T}_{\text {flat }}(M) \cong \mathbb{R}^{2}$. If $p>2$, then the angles in (9) are $\theta_{i}=\frac{2 \pi q_{i}}{p}$, for some $q_{i} \in \mathbb{Z}, i=1, \ldots, p-1$, with $q_{i} \not \equiv q_{j} \bmod p$. A special case is that of so-called generalized Klein bottles [10, § IV.9], which have dimension $p$, and holonomy $H_{\pi} \cong \mathbb{Z}_{p} \subset \mathrm{O}(p)$ generated by a matrix with characteristic polynomial $\lambda^{p}-1$. Thus, in this case $\theta_{i}=\frac{2 \pi i}{p}$ for each $i=1, \ldots, p-1$ and all $m_{i}=1$ except for $m_{2}=0$, so $l=\frac{1}{2}(p-1)$ and $\mathcal{T}_{\text {flat }}(M) \cong \mathbb{R}^{(p+1) / 2}$, cf. [10, Thm 9.1,p. 165].

\subsection{Flat 2-orbifolds}

As listed in Table 1, there are 17 affine equivalence classes of flat 2-orbifolds. Their Teichmüller spaces can be computed using Theorem B as follows:

(1) The Klein bottle, Möbius band, and cylinder have holonomy group $\mathbb{Z}_{2}$ generated by reflection about an axis and hence have 2 inequivalent 1-dimensional irreducibles. Thus, in these cases, $\mathcal{T}_{\text {flat }}(\mathcal{O})=\mathrm{GL}(1, \mathbb{R}) / \mathrm{O}(1) \times \mathrm{GL}(1, \mathbb{R}) / \mathrm{O}(1) \cong \mathbb{R}^{2}$. The case of the 2-torus is discussed in (8);

(2) The flat 2-orbifolds $D^{2}(; 3,3,3), D^{2}(; 2,3,6), D^{2}(; 2,4,4), D^{2}(4 ; 2), D^{2}(3 ; 3)$, $S^{2}(3,3,3 ;), S^{2}(2,3,6 ;)$, and $S^{2}(2,4,4 ;)$ have irreducible holonomy representation and hence $\mathcal{T}_{\text {flat }}(\mathcal{O})=\mathrm{GL}(1, \mathbb{R}) / \mathrm{O}(1) \cong \mathbb{R}$;

(3) The flat 2-orbifolds $D^{2}(; 2,2,2,2), D^{2}(2 ; 2,2), D^{2}(2,2 ;)$, and $\mathbb{R} P^{2}(2,2$; ) have holonomy group $D_{2} \cong \mathbb{Z}_{2} \oplus \mathbb{Z}_{2}$, generated by reflections about the coordinate axes and hence $\mathcal{T}_{\text {flat }}(\mathcal{O})=\mathrm{GL}(1, \mathbb{R}) / \mathrm{O}(1) \times \mathrm{GL}(1, \mathbb{R}) / \mathrm{O}(1) \cong \mathbb{R}^{2}$; 
(4) The flat 2-orbifold $S^{2}\left(2,2,2,2\right.$; ) has holonomy group $\mathbb{Z}_{2}$, generated by the rotation of $\pi$ and hence 1 isotypic component consisting of 2 copies of the nontrivial 1-dimensional representation. Thus, $\mathcal{T}_{\text {flat }}\left(S^{2}(2,2,2,2 ;)\right)=\mathrm{GL}(2, \mathbb{R}) / \mathrm{O}(2) \cong \mathbb{R}^{3}$.

\subsection{Flat 3-manifolds}

As mentioned in Sect. 2, there are 10 affine equivalence classes of closed flat 3-manifolds, described in Wolf [35, Thm. 3.5.5, 3.5.9]. These manifolds are labeled by the corresponding Bieberbach groups, denoted $\mathcal{G}_{i}, i=1, \ldots, 6$, in the orientable case, and $\mathcal{B}_{i}, i=1, \ldots, 4$, in the nonorientable case. Their Teichmüller space can be computed using Theorem B, reobtaining the results of $[24,25]$ :

(1) The flat 3-manifold corresponding to $\mathcal{G}_{1}$ is the 3-torus, see (8);

(2) The flat 3-manifolds corresponding to $\mathcal{G}_{3}, \mathcal{G}_{4}$, and $\mathcal{G}_{5}$ have holonomy isomorphic to $\mathbb{Z}_{k}$, with $k=3,4,6$, respectively, generated by a block diagonal matrix $A \in \mathrm{O}$ (3) with one eigenvalue 1 and a $2 \times 2$ block (9) with $\theta=\frac{2 \pi}{k}$. Thus, for these manifolds, $\mathcal{T}_{\text {flat }}(M) \cong \mathrm{GL}(1, \mathbb{R}) / \mathrm{O}(1) \times \mathrm{GL}(1, \mathbb{C}) / \mathrm{U}(1) \cong \mathbb{R}^{2}$;

(3) The flat 3-manifolds corresponding to $\mathcal{G}_{6}, \mathcal{B}_{3}$, and $\mathcal{B}_{4}$ have holonomy isomorphic to $\mathbb{Z}_{2} \oplus$ $\mathbb{Z}_{2}$, generated by $A_{1}=\operatorname{diag}(1,-1,1)$ and $A_{2}=\operatorname{diag}( \pm 1,1,-1)$. In all cases, there are 3 inequivalent 1-dimensional irreducibles. Thus, for such $M, \mathcal{I}_{\text {flat }}(M) \cong \mathrm{GL}(1, \mathbb{R}) / \mathrm{O}(1) \times$ $\mathrm{GL}(1, \mathbb{R}) / \mathrm{O}(1) \times \mathrm{GL}(1, \mathbb{R}) / \mathrm{O}(1) \cong \mathbb{R}^{3}$;

(4) The 3-manifolds corresponding to $\mathcal{G}_{2}, \mathcal{B}_{1}$, and $\mathcal{B}_{2}$ have holonomy isomorphic to $\mathbb{Z}_{2}$, generated by a diagonal matrix $A \in \mathrm{O}(3)$ with eigenvalues \pm 1 , one with multiplicity 1 and another with multiplicity 2 . Thus, the Teichmüller space of these manifolds is $\mathcal{T}_{\text {flat }}(M) \cong \mathrm{GL}(1, \mathbb{R}) / \mathrm{O}(1) \times \mathrm{GL}(2, \mathbb{R}) / \mathrm{O}(2) \cong \mathbb{R}^{4}$.

Comparing the above computations, it follows that closed flat 3-manifolds that have isomorphic holonomy groups also have diffeomorphic Teichmüller spaces. This coincidence, however, is of course not expected to hold in higher dimensions.

The computation of the groups $\mathcal{N}_{\pi}$ for the above manifolds, and hence of the moduli space of flat metrics $\mathcal{M}_{\text {flat }}(M)$, can be found in Kang [24, Thm. 4.5].

\subsection{Kummer surface}

The Kummer surface is given by $\mathcal{O}=T^{4} / \mathbb{Z}_{2}$, where $\mathbb{Z}_{2}$ acts via the antipodal map on each coordinate of $T^{4}$. This is a 4-dimensional flat orbifold with 16 conical singularities, whose desingularization is a Calabi-Yau $K 3$ surface (which is not flat, but admits Ricci flat metrics). The holonomy representation on $\mathbb{R}^{4}$ has one isotypic component consisting of 4 copies of the nontrivial $\mathbb{Z}_{2}$-representation. Thus, its Teichmüller space is $\mathcal{T}_{\text {flat }}(\mathcal{O}) \cong \mathrm{GL}(4, \mathbb{R}) / \mathrm{O}(4) \cong$ $\mathbb{R}^{10}$.

\subsection{Joyce orbifolds}

There are two interesting examples of 6-dimensional flat orbifolds that, similarly to the Kummer surface above, can be desingularized to Calabi-Yau manifolds as shown by Joyce [23]. The first, $\mathcal{O}_{1}=T^{6} / \mathbb{Z}_{4}$, also appears in the work of Vafa and Witten and has holonomy generated by the transformation $\operatorname{diag}(-1, i, i)$ of $\mathbb{C}^{3} \cong \mathbb{R}^{6}$. Thus, its Teichmüller space is $\mathcal{T}_{\text {flat }}\left(\mathcal{O}_{1}\right) \cong \mathrm{GL}(2, \mathbb{R}) / \mathrm{O}(2) \times \mathrm{GL}(2, \mathbb{C}) / \mathrm{U}(2) \cong \mathbb{R}^{7}$. The second, $\mathcal{O}_{2}=T^{6} / \mathbb{Z}_{2} \oplus \mathbb{Z}_{2}$, has holonomy generated by the transformations $\operatorname{diag}(1,-1,-1)$ and $\operatorname{diag}(-1,1,-1)$ of $\mathbb{C}^{3} \cong \mathbb{R}^{6}$, and thus, $\mathcal{T}_{\text {flat }}\left(\mathcal{O}_{2}\right) \cong \mathrm{GL}(2, \mathbb{R}) / \mathrm{O}(2) \times \mathrm{GL}(2, \mathbb{R}) / \mathrm{O}(2) \times \mathrm{GL}(2, \mathbb{R}) / \mathrm{O}(2) \cong \mathbb{R}^{9}$. 


\section{Classification of collapsed limits of flat 3-manifolds}

In this section, we analyze the collapsed limits of closed flat 3-manifolds to prove Theorem D.

For completeness, let us briefly discuss the trivial situation of collapse of flat manifolds in dimensions $<3$. In dimension 1 , the only closed (flat) manifold is $S^{1}$, and its Teichmüller space is clearly 1-dimensional. In this case, the only possible collapse is to a point.

The 2-dimensional closed flat manifolds are the 2-torus $T^{2}$ and the Klein bottle $K^{2}$. From Proposition 3.1, the only possible collapsed limits of $T^{2}$ are a point or a circle. The Bieberbach group $\pi$ corresponding to the Klein bottle $K^{2}=\mathbb{R}^{2} / \pi$ is generated by a lattice $L_{\pi}$, whose basis $\left\{v_{1}, v_{2}\right\}$ consists of orthogonal vectors, and $\left(A, \frac{1}{2} v_{1}\right)$, where $A$ is the reflection about the line spanned by $v_{1}$. Thus, $H_{\pi} \cong \mathbb{Z}_{2}$ has isotypic components $W_{1}=\operatorname{span}\left\{v_{1}\right\}$ and $W_{2}=\operatorname{span}\left\{v_{2}\right\}$, which are the only two possible directions along which flat metrics on $K^{2}$ can collapse. The limit obtained by collapsing $W_{2}$ is clearly $S^{1}$. By Proposition 3.2, the limit obtained by collapsing $W_{1}$ is the orbit space of the reflection $\mathbb{Z}_{2}$-action on the circle $S^{1}$, which is a closed interval. Therefore, $K^{2}$ can collapse to a point, to a circle, or to a closed interval. Geometrically, these can be seen as shrinking the lengths of either pair of opposite sides of the rectangle with boundary identifications that customarily represents $K^{2}$.

To analyze the possible collapses of closed flat 3-manifolds, we proceed case by case, in the same order as in Sect. 5.4, following the notation of Wolf [35, Thm. 3.5.5]. The first (trivial) case $\pi=\mathcal{G}_{1}$ is that of the 3 -torus $T^{3}$, which by Proposition 3.1 can only collapse to a point, to $S^{1}$, or to $T^{2}$. In the remaining cases $\mathbb{R}^{3} / \pi$, we denote by $\left\{v_{1}, v_{2}, v_{3}\right\}$ a basis of the lattice $L_{\pi}$ and identify each vector $v \in L_{\pi}$ with (Id, $\left.v\right) \in \pi$. Henceforth, we ignore the case of iterated collapses, that is, if a flat manifold $M$ collapses to a flat orbifold $\mathcal{O}_{1}$ and this orbifold collapses to another flat orbifold $\mathcal{O}_{2}$, then clearly $M$ can be collapsed (directly) to $\mathrm{O}_{2}$.

\subsection{Cases with 2-dimensional Teichmüller space}

There are three flat 3-manifolds with 2-dimensional Teichmüller space; namely those with Bieberbach groups $\mathcal{G}_{3}, \mathcal{G}_{4}$, and $\mathcal{G}_{5}$, see Sect. 5.4(2).

Example 6.1 (Case $\mathcal{G}_{3}$ ) The basis of the lattice $L_{\pi}$ of the Bieberbach group $\pi=\mathcal{G}_{3}$ is such that $v_{1}$ is orthogonal to both $v_{2}$ and $v_{3},\left\|v_{2}\right\|=\left\|v_{3}\right\|$, and $v_{2}$ and $v_{3}$ span a 2-dimensional hexagonal lattice. This group $\pi$ is generated by $L_{\pi}$ and $\left(A, \frac{1}{3} v_{1}\right)$, where $A$ fixes $v_{1}$ and rotates its orthogonal complement by $\frac{2 \pi}{3}$. Thus, $H_{\pi} \cong \mathbb{Z}_{3}$ and its two isotypic components are $W_{1}=\operatorname{span}\left\{v_{1}\right\}$ and $W_{2}=\operatorname{span}\left\{v_{2}, v_{3}\right\}$. Collapsing $W_{2}$, the limit is clearly $S^{1}$. From Proposition 3.2, the limit obtained by collapsing $W_{1}$ is the orbit space of a $\mathbb{Z}_{3}$-action on the flat 2-torus given by the quotient of $W_{2} \cong \mathbb{R}^{2}$ by the hexagonal lattice. A generator of $\mathbb{Z}_{3}$ acts on $W_{2}$ by (clockwise) rotation of angle $\frac{2 \pi}{3}$, which leaves invariant the hexagonal lattice, and hence descends to the relevant $\mathbb{Z}_{3}$-action on $T^{2}$. The parallelogram in $W_{2}$ with vertices $0, v_{2}, v_{3}$, and $v_{2}+v_{3}$ is a fundamental domain for this action. The orbit space $T^{2} / \mathbb{Z}_{3}$ is easily identified as the 2-orbifold $S^{2}(3,3,3 ;)$ by analyzing the boundary identifications induced on this fundamental domain.

Example 6.2 (Case $\mathcal{G}_{4}$ ) The basis of the lattice $L_{\pi}$ of the Bieberbach group $\pi=\mathcal{G}_{4}$ consists of pairwise orthogonal vectors $v_{1}, v_{2}$, and $v_{3}$, with $\left\|v_{2}\right\|=\left\|v_{3}\right\|$. This group $\pi$ is generated by $L_{\pi}$ and $\left(A, \frac{1}{4} v_{1}\right)$, where $A$ fixes $v_{1}$ and rotates its orthogonal complement by $\frac{\pi}{2}$. Thus, $H_{\pi} \cong \mathbb{Z}_{4}$ and the two isotypic components are $W_{1}=\operatorname{span}\left\{v_{1}\right\}$ and $W_{2}=\operatorname{span}\left\{v_{2}, v_{3}\right\}$. The limit obtained by collapsing $W_{2}$ is clearly $S^{1}$. Collapsing $W_{1}$, the limit is the orbit space 
of a $\mathbb{Z}_{4}$-action on the flat 2-torus given by the quotient of $W_{2} \cong \mathbb{R}^{2}$ by the square lattice generated by $v_{2}$ and $v_{3}$. A generator of $\mathbb{Z}_{4}$ acts on $W_{2}$ by (clockwise) rotation of angle $\frac{\pi}{2}$, which leaves invariant the square lattice, and hence descends to the relevant $\mathbb{Z}_{4}$-action on $T^{2}$. The square with vertices $0, v_{2}, v_{3}$, and $v_{2}+v_{3}$ is a fundamental domain for this action, which is generated by the rotation of angle $\frac{\pi}{4}$ around its center. The orbit space $T^{2} / \mathbb{Z}_{4}$ is identified with $D^{2}(4 ; 2)$, which has a singular point on the boundary corresponding to the orbit of the vertices of the square, and an interior singular point corresponding to the center of the square which is fixed by $\mathbb{Z}_{4}$.

Example 6.3 (Case $\mathcal{G}_{5}$ ) The Bieberbach group $\pi=\mathcal{G}_{5}$ is generated by the same lattice $L_{\pi}$ as in Example 6.1 and $\left(A, \frac{1}{6} v_{3}\right)$, where $A$ fixes $v_{1}$ and rotates its orthogonal complement by $\frac{\pi}{3}$. Thus, $H_{\pi} \cong \mathbb{Z}_{6}$ and the two isotypic components are again $W_{1}=\operatorname{span}\left\{v_{1}\right\}$ and $W_{2}=\operatorname{span}\left\{v_{2}, v_{3}\right\}$. The limit obtained collapsing $W_{2}$ is clearly $S^{1}$. Collapsing $W_{1}$, the limit is the orbit space of a $\mathbb{Z}_{6}$-action on the flat 2-torus given by the quotient of $W_{2} \cong \mathbb{R}^{2}$ by the hexagonal lattice. A generator of $\mathbb{Z}_{6}$ acts on $W_{2}$ by (clockwise) rotation of angle $\frac{\pi}{3}$, which leaves invariant the hexagonal lattice, and hence descends to the relevant $\mathbb{Z}_{6}$ action on $T^{2}$. The equilateral triangle in $W_{2}$ with vertices $0, v_{2}$, and $v_{3}$ is a fundamental domain for this action, which is generated by the rotation of angle $\frac{\pi}{3}$ around its center. Therefore, the orbit space $T^{2} / \mathbb{Z}_{6}$ is identified with $D^{2}(3 ; 3)$, which has a singular point on the boundary corresponding to the orbit of the vertices of the triangle, and an interior singular point corresponding to the center of the triangle which is fixed by $\mathbb{Z}_{6}$. Alternatively, this orbifold can be seen as the quotient of $S^{2}(3,3,3$; ) by an involution given by reflection about the equator through one of the singular points. In fact, the subaction of $\mathbb{Z}_{3} \triangleleft \mathbb{Z}_{6}$ on $T^{2}$ has orbit space $T^{2} / \mathbb{Z}_{3}=S^{2}(3,3,3 ;)$ as described in Example 6.1, and hence, the orbit space $T^{2} / \mathbb{Z}_{6}$ is given by $S^{2}(3,3,3 ;) / \mathbb{Z}_{2}=D^{2}(3 ; 3)$.

Note that the flat 2-orbifolds $S^{2}(3,3,3 ;), D^{2}(4 ; 2)$, and $D^{2}(3 ; 3)$ that arise as collapsed limits of the above flat 3-manifolds $M$ with 2-dimensional Teichmüller space have irreducible holonomy (see Sect. 5.3). Thus, the Teichmüller space of such orbifolds is 1-dimensional. This is in accordance with the stratification of the ideal boundary of $\mathcal{T}_{\text {flat }}(M) \cong \mathbb{R}^{2}$ by the Teichmüller spaces of the collapsed limits of $M$, which also include a point and $S^{1}$ that have 0- and 1-dimensional Teichmüller spaces, respectively.

Remark 6.4 Similar conclusions regarding the ideal boundary of Teichmüller spaces hold in general, for instance, in the higher dimensional cases discussed below, provided that two subtleties are taken into account. First, if a flat orbifold $\mathcal{O}$ is a collapsed limit of a flat manifold $M$, it is not necessarily true that all flat metrics on $\mathcal{O}$ arise as collapsed limits of flat metrics on $M$. Thus, parts of the Teichmüller space of $\mathcal{O}$ might be absent from the stratum of the boundary of $\mathcal{T}_{\text {flat }}(M)$ that corresponds to collapse to $\mathcal{O}$. Second, there may be a continuum of inequivalent ways to collapse $M$ to $\mathcal{O}$, so that the stratum of the boundary of $\mathcal{T}_{\text {flat }}(M)$ that corresponds to collapse to $\mathcal{O}$ may contain a continuum of copies of $\mathcal{T}_{\text {flat }}(O)$.

Recall that the Gromov-Hausdorff distance between the metric spaces given by $M$ equipped with different flat metrics does not extend continuously to the ideal boundary of $\mathcal{T}_{\text {flat }}(M)$, as discussed in the Introduction.

\subsection{Cases with 3-dimensional Teichmüller space}

There are three flat 3-manifolds with 3-dimensional Teichmüller space, namely those with Bieberbach groups $\mathcal{G}_{6}, \mathcal{B}_{3}$, and $\mathcal{B}_{4}$, see Sect. 5.4(3). 
Example 6.5 (Case $\mathcal{G}_{6}$ ) The basis of the lattice $L_{\pi}$ of the Bieberbach group $\pi=\mathcal{G}_{6}$ consists of pairwise orthogonal vectors $v_{1}, v_{2}$ and $v_{3}$. This group $\pi$ is generated by $L_{\pi}$ together with

$$
\left(A, \frac{1}{2} v_{1}\right), \quad\left(B, \frac{1}{2}\left(v_{1}+v_{2}\right)\right), \quad\left(C, \frac{1}{2}\left(v_{1}+v_{2}+v_{3}\right)\right),
$$

where $A=\operatorname{diag}(1,-1,-1), B=\operatorname{diag}(-1,1,-1)$, and $C=\operatorname{diag}(-1,-1,1)$ in the basis $\left\{v_{1}, v_{2}, v_{3}\right\}$. Thus, $H_{\pi} \cong \mathbb{Z}_{2} \oplus \mathbb{Z}_{2}$, and the three isotypic components are $W_{i}=\operatorname{span}\left\{v_{i}\right\}$, $i=1,2,3$.

Case (a) Collapsing $W_{1}$ yields a flat orbifold obtained as the quotient of $\mathbb{R}^{2}$ by the action of the group of isometries generated by $T_{1}(x, y)=(x+a, y), T_{2}(x, y)=(x, y+b)$, $T_{3}(x, y)=(-x,-y), T_{4}(x, y)=\left(x+\frac{1}{2} a,-y+\frac{1}{2} b\right)$ and $T_{5}(x, y)=(-x+$ $\left.\frac{1}{2} a, y+\frac{1}{2} b\right)$, where $a=\left|v_{2}\right|>0$ and $b=\left|v_{3}\right|>0$. This orbifold can be identified with $\mathbb{R} P^{2}(2,2 ;)$.

Case (b) Collapsing $W_{2}$, we obtain the flat orbifold given by the quotient of $\mathbb{R}^{2}$ by the group of isometries generated by $T_{1}(x, y)=(x+a, y), T_{2}(x, y)=(x, y+b), T_{3}(x, y)=$ $\left(x+\frac{1}{2} a,-y\right), T_{4}(x, y)=\left(-x,-y+\frac{1}{2} b\right)$ and $T_{5}(x, y)=\left(-x+\frac{1}{2} a, y+\frac{1}{2} b\right)$, where $a=\left|v_{1}\right|>0$ and $b=\left|v_{3}\right|>0$. Clearly, $T_{3}^{2}=T_{1}$. As above, this can be identified with $\mathbb{R} P^{2}(2,2 ;)$.

Case (c) Collapsing $W_{3}$, we obtain the flat orbifold given by the quotient of $\mathbb{R}^{2}$ by the group of isometries generated by $T_{1}(x, y)=(x+a, y), T_{2}(x, y)=(x, y+b)$, $T_{3}(x, y)=\left(x+\frac{1}{2} a,-y\right), T_{4}(x, y)=\left(-x, y+\frac{1}{2} b\right)$ and $T_{5}=\left(-x+\frac{1}{2} a,-y+\frac{1}{2} b\right)$, where $a=\left|v_{1}\right|>0$ and $b=\left|v_{2}\right|>0$. Clearly, $T_{1}=T_{3}^{2}, T_{2}=T_{4}^{2}$ and $T_{4}=T_{5} \circ T_{3}$. As above, this can be identified with $\mathbb{R} P^{2}(2,2 ;)$.

Example 6.6 (Case $\mathcal{B}_{3}$ ) The basis of the lattice $L_{\pi}$ of the Bieberbach group $\pi=\mathcal{B}_{3}$ consists of pairwise orthogonal vectors. The group $\pi$ is generated by $L_{\pi}$ together with $\left(A, \frac{1}{2} v_{1}\right)$ and $\left(E, \frac{1}{2} v_{2}\right)$, where $A=\operatorname{diag}(1,-1,-1)$ and $E=\operatorname{diag}(1,1,-1)$ in the basis $\left\{v_{1}, v_{2}, v_{3}\right\}$. Thus, $H_{\pi} \cong \mathbb{Z}_{2} \times \mathbb{Z}_{2}$, and the three isotypic components are $W_{i}=\operatorname{span}\left\{v_{i}\right\}, i=1,2,3$.

Case (a) Collapsing $W_{1}$ yields a flat orbifold obtained as the quotient of $\mathbb{R}^{2}$ by the action of the group of isometries generated by $T_{1}(x, y)=(x+a, y), T_{2}(x, y)=(x, y+b)$, $T_{3}(x, y)=(-x,-y)$ and $T_{4}(x, y)=\left(x+\frac{1}{2} a,-y\right)$, where $a=\left|v_{2}\right|>0$ and $b=\left|v_{3}\right|>0$. This orbifold is $D^{2}(2,2 ;)$.

Case (b) Collapsing $W_{2}$ yields a flat orbifold obtained as the quotient of $\mathbb{R}^{2}$ by the action of the group of isometries generated by $T_{1}(x, y)=(x+a, y), T_{2}(x, y)=(x, y+b)$, $T_{3}(x, y)=(x,-y)$ and $T_{4}(x, y)=\left(x+\frac{1}{2} a,-y\right)$, where, $a=\left|v_{1}\right|>0$ and $b=\left|v_{3}\right|>0$. This orbifold is $S^{1} \times I$.

Case (c) Collapsing $W_{3}$ yields a flat orbifold obtained as the quotient of $\mathbb{R}^{2}$ by the action of the group of isometries generated by $T_{1}(x, y)=(x+a, y), T_{2}(x, y)=(x, y+b)$, $T_{3}(x, y)=\left(x+\frac{1}{2} a,-y\right)$ and $T_{4}(x, y)=\left(x, y+\frac{1}{2} b\right)$. Here, $a=\left|v_{1}\right|>0$ and $b=\left|v_{2}\right|>0$. Clearly, $T_{1}=T_{3}^{2}$ and $T_{2}=T_{4}^{2}$. This orbifold is easily identified with a flat Klein bottle.

Example 6.7 (Case $\left.\mathcal{B}_{4}\right)$ The basis of the lattice $L_{\pi}$ of the Bieberbach group $\pi=\mathcal{B}_{4}$ also consists of pairwise orthogonal vectors. The group $\pi$ is generated by $L_{\pi}$ together with $\left(A, \frac{1}{2} v_{1}\right)$ and $\left(E, \frac{1}{2}\left(v_{2}+v_{3}\right)\right)$, where $A=\operatorname{diag}(1,-1,-1)$ and $E=\operatorname{diag}(1,1,-1)$ in the basis $\left\{v_{1}, v_{2}, v_{3}\right\}$. Thus, $H_{\pi} \cong \mathbb{Z}_{2} \times \mathbb{Z}_{2}$, and the three isotypic components are $W_{i}=$ $\operatorname{span}\left\{v_{i}\right\}, i=1,2,3$.

Case (a) Collapsing $W_{1}$ yields a flat orbifold obtained as the quotient of $\mathbb{R}^{2}$ by the action of the group of isometries generated by $T_{1}(x, y)=(x+a, y), T_{2}(x, y)=(x, y+b)$, 
$T_{3}(x, y)=(-x,-y)$ and $T_{4}(x, y)=\left(x+\frac{1}{2} a, y+\frac{1}{2} b\right)$, where, $a=\left|v_{2}\right|>0$ and $b=\left|v_{3}\right|>0$. This orbifold is $S^{2}(2,2,2,2 ;)$.

Case (b) Collapsing $W_{2}$ yields a flat orbifold obtained as the quotient of $\mathbb{R}^{2}$ by the action of the group of isometries generated by $T_{1}(x, y)=(x+a, y), T_{2}(x, y)=(x, y+b)$, $T_{3}(x, y)=\left(x,-y+\frac{1}{2} b\right)$ and $T_{4}(x, y)=\left(x+\frac{1}{2} a,-y\right)$, where $a=\left|v_{1}\right|>0$ and $b=\left|v_{3}\right|>0$. This orbifold is a Möbius band.

Case (c) Collapsing $W_{3}$ yields a flat orbifold obtained as the quotient of $\mathbb{R}^{2}$ by the action of the group of isometries generated by $T_{1}(x, y)=(x+a, y), T_{2}(x, y)=(x, y+b)$, $T_{3}(x, y)=\left(x, y+\frac{1}{2} b\right)$ and $T_{4}(x, y)=\left(x+\frac{1}{2} a,-y\right)$, where $a=\left|v_{1}\right|>0$ and $b=\left|v_{2}\right|>0$. Clearly, $T_{4}^{2}=T_{1}$ and $T_{3}^{2}=T_{2}$. This orbifold is easily identified with a Klein bottle.

\subsection{Cases with 4-dimensional Teichmüller space}

There are three flat 3-manifolds with 4-dimensional Teichmüller space; namely those with Bieberbach groups $\mathcal{B}_{1}, \mathcal{B}_{2}$, and $\mathcal{G}_{2}$, see Sect. 5.4(4).

Example 6.8 (Case $\left.\mathcal{B}_{1}\right)$ The manifold in question is the product $K^{2} \times S^{1}$, which by the discussion in the beginning of this section can collapse to $K^{2}, S^{1} \times I, I, S^{1}$, and a point.

Example 6.9 (Case $\mathcal{B}_{2}$ ) The basis of the lattice $L_{\pi}$ of the Bieberbach group $\pi=\mathcal{B}_{2}$ is such that $v_{1}$ and $v_{2}$ generate any planar lattice, while $v_{3}$ is a vector whose orthogonal projection on the plane spanned by $v_{1}$ and $v_{2}$ is $\frac{1}{2}\left(v_{1}+v_{2}\right)$. This group $\pi$ is generated by $L_{\pi}$ and $\left(E, \frac{1}{2} v_{1}\right)$, where $E$ is the identity on the plane spanned by $v_{1}$ and $v_{2}$, and $E\left(v_{3}\right)=v_{1}+v_{2}-v_{3}$, i.e., $E=\operatorname{diag}(1,1,-1)$ in the basis $\left\{v_{1}, v_{2}, w\right\}$, where $w$ is orthogonal to $v_{1}$ and $v_{2}$. Thus, $H_{\pi} \cong \mathbb{Z}_{2}$ and it has one trivial isotypic component $W_{1}=\operatorname{span}\left\{v_{1}, v_{2}\right\}$ and one nontrivial isotypic component $W_{2}=\operatorname{span}\{w\}$ isomorphic to the sign representation of $\mathbb{Z}_{2}$.

Collapsing $W_{2}$, the limit is clearly $T^{2}$. Collapsing $W_{1}$, the limit is an interval $I$. Collapsing a one-dimensional subspace of $W_{1}$ produces as limit either a Möbius band, a Klein bottle, or an interval, depending on the slope of the subspace. More precisely, collapsing the direction of $v_{1}$, the limiting orbifold is the quotient of $\mathbb{R}^{2}$ by the group of isometries generated by $T_{1}(x, y)=(x+a, y), T_{2}(x, y)=\left(x+\frac{1}{2} a, y+b\right)$, and $T_{3}(x, y)=(x,-y)$. Here, $a=$ $\left|v_{2}\right|>0$ and $b=\left|v_{3}\right|>0$. This orbifold can be identified with a Möbius band. On the other hand, collapsing the direction of $v_{2}$, the limiting orbifold is the quotient of $\mathbb{R}^{2}$ by the group of isometries generated by $T_{1}(x, y)=(x+a, y), T_{2}(x, y)=\left(x+\frac{1}{2} a, y+b\right)$, and $T_{3}(x, y)=\left(x+\frac{1}{2} a,-y\right)$. Here, $a=\left|v_{1}\right|>0$ and $b=\left|v_{3}\right|>0$. Clearly, $T_{3}^{2}=T_{1}$, so that $T_{1}$ can be omitted from the list of generators. This quotient can be identified with a Klein bottle. Collapsing (generic) directions with irrational slope with respect to $v_{1}$ and $v_{2}$ has the same effect as collapsing all of $W_{1}$, which results in an interval as the limit.

Example 6.10 (Case $\mathcal{G}_{2}$ ) The basis of the lattice $L_{\pi}$ of the Bieberbach group $\pi=\mathcal{G}_{2}$ is such that $v_{1}$ is orthogonal to $v_{2}$ and $v_{3}$. This group $\pi$ is generated by $L_{\pi}$ and $\left(A, \frac{1}{2} v_{1}\right)$, where $A\left(v_{1}\right)=v_{1}$, and $A(v)=-v$ for all $v$ in the span of $v_{2}$ and $v_{3}$. Thus, $H_{\pi} \cong \mathbb{Z}_{2}$ and it has one trivial isotypic component $W_{1}=\operatorname{span}\left\{v_{1}\right\}$ and one nontrivial isotypic component $W_{2}=\operatorname{span}\left\{v_{2}, v_{3}\right\}$, isomorphic to the direct sum of two copies of the sign representation of $\mathbb{Z}_{2}$. Collapsing $W_{1}$, the limit is the quotient of a $\mathbb{R}^{2}$ by the group of isometries generated by $T_{1}, T_{2}, T_{3}$ and $T_{4}=-\mathrm{Id}$, with $T_{1}(x, y)=(x+a, y), T_{2}(x, y)=(x, y+b)$, and $T_{3}(x, y)=\left(x+\frac{a}{2}, y\right)$. Here, $a=\left|v_{1}\right|$ and $b=|v|$. Clearly, $T_{1}=T_{3}^{2}$. A fundamental domain for this quotient is given by the rectangular triangle with vertices $(0,0),(a, 0)$ and $(0, b)$. This limit can be identified with the flat 2-orbifold $D^{2}\left(2,2\right.$; ). Collapsing $W_{2}$, the limit is 
clearly $S^{1}$. Collapsing a one-dimensional subspace of $W_{2}$ produces as limit either a flat Klein bottle or a point, depending on the slope of the subspace.

Acknowledgements It is a pleasure to thank Alexander Lytchak, John Harvey, Karsten Grove, and Curtis Pro for suggestions regarding equivariant Gromov-Hausdorff convergence, Burkhard Wilking for comments on realizing flat orbifolds as limits of flat manifolds, and Andrzej Szczepański for conversations about the holonomy representation of flat manifolds. Part of this work was done during a visit of the first named author to the Max Planck Institute for Mathematics in Bonn, Germany, and of the third named author to the University of Notre Dame, USA; they would like to thank these institutions for providing excellent working conditions. The second and third named authors are partially supported by a FAPESP-OSU 2015 Regular Research Award (FAPESP Grant: 2015/50315-3).

\section{References}

1. Alexandrino, M.M., Bettiol, R.G.: Lie Groups and Geometric Aspects of Isometric Actions. Springer, New York (2015)

2. Auslander, L., Kuranishi, M.: On the holonomy group of locally Euclidean spaces. Ann. Math. 65(2), 411-415 (1957)

3. Baues, O.: Varieties of discontinuous groups. In: Crystallographic Groups and Their Generalizations (Kortrijk, 1999), vol. 262 of Contemporary Mathematics. American Mathematical Society, Providence, pp. 147-158 (2000)

4. Bettiol, R.G., Piccione, P.: Infinitely many solutions to the Yamabe problem on noncompact manifolds. Ann. Inst. Fourier (Grenoble) (to appear). arXiv:1603.07788

5. Bieberbach, L.: Über die Bewegungsgruppen der Euklidischen Räume, I. Math. Ann. 70, 297-336 (1911)

6. Bieberbach, L.: Über die Bewegungsgruppen der Euklidischen Räume, II. Die Gruppen mit einem endlichen Fundamentalbereich. Math. Ann. 72, 400-412 (1912)

7. Bridson, M.R., Haefliger, A.: Metric Spaces of Non-positive Curvature Vol. 319 of Grundlehren der Mathematischen Wissenschaften [Fundamental Principles of Mathematical Sciences]. Springer, Berlin (1999)

8. Brown, H., Bülow, R., Neubüser, J., Wondratschek, H., Zassenhaus, H.: Crystallographic Groups of Four-dimensional Space, Wiley Monographs in Crystallography. Wiley, New York (1978)

9. Buser, P.: A geometric proof of Bieberbach's theorems on crystallographic groups. Enseign. Math. (2) 31, 137-145 (1985)

10. Charlap, L.S.: Bieberbach Groups and Flat Manifolds. Universitext, Springer, New York (1986)

11. Cid, C., Schulz, T.: Computation of five- and six-dimensional Bieberbach groups. Exp. Math. 10, 109-115 (2001)

12. Conway, J.H.: The orbifold notation for surface groups. In: Groups, Combinatorics and Geometry (Durham, 1990), vol. 165 of London Mathematical Society Lecture Note Series. Cambridge University Press, Cambridge, pp. 438-447 (1992)

13. Davis, M.W.: Lectures on orbifolds and reflection groups. In: Transformation Groups and Moduli Spaces of Curves, Volume 16 of Advanced Lectures in Mathematics (ALM). International Press, Somerville, pp. 63-93 (2011)

14. Farb, B., Margalit, D.: A Primer on Mapping Class Groups. Princeton Mathematical Series, vol. 49. Princeton University Press, Princeton (2012)

15. Fukaya, K.: Theory of convergence for Riemannian orbifolds. Jpn. J. Math. (N.S.) 12, 121-160 (1986)

16. Fukaya, K., Yamaguchi, T.: The fundamental groups of almost non-negatively curved manifolds. Ann. Math. (2) 136, 253-333 (1992)

17. Galaz-Garcia, F., Guijarro, L.: On Three-Dimensional Alexandrov Spaces. International Mathematics Research Notices IMRN, pp. 5560-5576 (2015)

18. Goldman, W.M.: Geometric structures on manifolds and varieties of representations. In: Geometry of Group Representations (Boulder, CO, 1987), Volume 74 of Contemporary Mathematics. American Mathematical Society, Providence, pp. 169-198 (1988)

19. Gromov, M.: Metric structures for Riemannian and non-Riemannian spaces. Modern Birkhäuser Classics, Birkhäuser Boston, Inc., Boston, English ed., Based on the 1981 French original, With appendices by M. Katz, P. Pansu and S. Semmes (2007)

20. Hantzsche, W., Wendt, H.: Dreidimensionale euklidische Raumformen. Math. Ann. 110, 593-611 (1935)

21. Harvey, J., Searle, C.: Orientation and symmetries of Alexandrov spaces with applications in positive curvature. J. Geom. Anal. 27(2), 1636-1666 (2017) 
22. Hiss, G., Szczepański, A.: On torsion free crystallographic groups. J. Pure Appl. Algebra 74, 39-56 (1991)

23. Joyce, D.: Deforming Calabi-Yau orbifolds. Asian J. Math. 3, 853-867 (1999)

24. Kang, E.S.: Moduli spaces of 3-dimensional flat manifolds. J. Korean Math. Soc. 43, 1065-1080 (2006)

25. Kang, E.S., Kim, J.Y.: Deformation spaces of 3-dimensional flat manifolds. Commun. Korean Math. Soc. 18, 95-104 (2003)

26. Kleiner, B., Lott, J.: Geometrization of three-dimensional orbifolds via Ricci flow. Astérisque No. 365, 101-177 (2014)

27. Mahler, K.: On lattice points in $n$-dimensional star bodies. I. Existence theorems. Proc. R. Soc. Lond. Ser. A 187, 151-187 (1946)

28. Matsumoto, Y., Montesinos-Amilibia, J.M.: A proof of Thurston's uniformization theorem of geometric orbifolds. Tokyo J. Math. 14, 181-196 (1991)

29. Pansu, P.: Effondrement des variétés riemanniennes, d'après J. Cheeger et M. Gromov, Astérisque. Seminar Bourbaki, vol. 1983/84, pp. 63-82 (1985)

30. Plesken, W., Schulz, T.: Counting crystallographic groups in low dimensions. Exp. Math. 9, 407-411 (2000)

31. Szczepański, A.: Problems on Bieberbach groups and flat manifolds. Geom. Dedicata 120, 111-118 (2006)

32. Szczepański, A.: Geometry of Crystallographic Groups, Volume 4 of Algebra and Discrete Mathematics. World Scientific Publishing Co. Pte. Ltd., Hackensack (2012)

33. Thurston, W.P.: Three-dimensional geometry and topology. In: Silvio, L. (ed.) Volume 35 of Princeton Mathematical Series, vol. 1. Princeton University Press, Princeton (1997)

34. Wolf, J.A.: Local and global equivalence for flat affine manifolds with parallel geometric structures. Geom. Dedicata 2, 127-132 (1973)

35. Wolf, J.A.: Spaces of Constant Curvature, 5th edn. Publish or Perish Inc, Houston (1984) 